Review Article

\title{
The Epidemiology and Economic Burden of Obesity and Related Cardiometabolic Disorders in the United Arab Emirates: A Systematic Review and Qualitative Synthesis
}

\author{
Hadia Radwan $\left(\mathbb{D},{ }^{1}\right.$ Rami A. Ballout ${ }^{D},{ }^{2}$ Hayder Hasan $\left(\mathbb{D},{ }^{1}\right.$ Nader Lessan, ${ }^{3}$ \\ Mirey Karavetian $\left(\mathbb{B},{ }^{4}\right.$ and Rana Rizk $\mathbb{1}^{5,6}$ \\ ${ }^{1}$ Department of Clinical Nutrition and Dietetics, College of Health Sciences, \\ Research Institute of Medical \& Health Sciences (RIMHS), University of Sharjah, P.O. Box 27272, Sharjah, UAE \\ ${ }^{2}$ Faculty of Medicine, American University of Beirut, Beirut, Lebanon \\ ${ }^{3}$ Imperial College London Diabetes Centre, P.O. Box 48338, Abu Dhabi, UAE \\ ${ }^{4}$ Department of Health Sciences, College of Natural and Health Sciences, Zayed University, P.O. Box 144534, Dubai, UAE \\ ${ }^{5}$ INSPECT-LB, Institut National de Santé Publique, d'Épidémiologie Clinique et de Toxicologie, Faculty of Public Health, \\ The Lebanese University, Beirut, Lebanon \\ ${ }^{6}$ Department of Health Services Research, Maastricht University, 6200 MD Maastricht, Netherlands
}

Correspondence should be addressed to Rana Rizk; r.rizk@maastrichtuniversity.nl

Hadia Radwan and Rami A. Ballout are co-first authors.

Received 24 August 2018; Accepted 30 September 2018; Published 3 December 2018

Academic Editor: Eliot Brinton

Copyright ( $\odot 2018$ Hadia Radwan et al. This is an open access article distributed under the Creative Commons Attribution License, which permits unrestricted use, distribution, and reproduction in any medium, provided the original work is properly cited.

Background. Noncommunicable diseases (NCDs) are considered as a global health problem and considered as a public health priority with the more considerable increasing trend of obesity and cardiometabolic disorders rates in the Middle Eastern countries. This systematic review aims at assessing the prevalence, incidence rates, and trends, as well as the cost of obesity and related cardiometabolic disorders in the United Arab Emirates (UAE). Methods. A highly sensitive strategy was used to retrieve original observational studies, addressing the epidemiology and cost of obesity and related cardiometabolic disorders in the UAE, irrespective of nationality (nationals and expatriates). The search was conducted on April 4, 2017, within numerous electronic databases and the grey literature. Standardized and validated methods were used for data extraction and analysis as well as quality assessment. Results. 6789 records were retrieved, of which 36 were deemed eligible. High prevalence rates were reported for obesity, diabetes, hypertension, and metabolic syndrome in all studies. However, the definitions and methods employed by the studies were highly variable. The risk of bias in the epidemiological studies ranged between low and medium. Only one study reported the cost of illness for diabetes. In this study, the estimated cost per patient was \$2,015 (adjusted to the year 2015), and it became twofold and sixfold higher in patients with microvascular and macrovascular complications, respectively. Conclusions. Obesity and related cardiometabolic disorders are highly prevalent in the UAE, but quoting a precise prevalence for them is difficult given the methodological heterogeneity of the epidemiological studies addressing them. Nonetheless, we detected a 2-3-fold increase in the prevalence of overweight and obesity in the UAE between 1989 and 2017. It is hopeful that this systematic review will provide an insight into direct future studies, especially longitudinal studies exploring obesity and cardiometabolic risks and their costs.

\section{Introduction}

The global rise in the prevalence rates of obesity, metabolic syndrome, and diabetes has been linked to recent lifestyle changes occurring during the past few decades, with the
Middle East in general and the United Arab Emirates (UAE) in particular demonstrating dramatic increases in those rates [1]. The UAE has witnessed an economic boom that was accompanied by rapid urbanization and an influx of expatriate workforce [1]. The combination of these factors 
meant a modern, fast-paced, and technology-driven lifestyle which, in turn, resulted in a reduction in occupational, domestic, and leisure-time physical activity $[2,3]$, as well as an excessive consumption of calorie-dense, processed, and prepackaged meals of poor nutritional value [3].

The abovementioned risk factors, in addition to the high incidence of tobacco use in the UAE, possibly explain the witnessed increase in obesity and cardiometabolic disorder rates in the country $[4,5]$, resulting in increased demand for disease-specific health services. A recent systematic review on the UAE's health status ranks cardiovascular diseases as a top public health priority for the country, attributing to it the majority of noncommunicable disease- (NCD-) related mortalities [6]. This overlaps with the global epidemiology of NCDs, among which cardiovascular diseases also rank first in terms of incidence and mortality [7]. In fact, the UAE's 2021 vision is to adopt the number of deaths due to cardiovascular diseases per 100,000 population, the prevalence of diabetes, and the prevalence of obesity amongst children as its primary national performance indicators for a desired world-class health care, in order to guide targeted interventions and public health efforts [8].

Therefore, the following systematic review provides a current evidence-based assessment of the epidemiology and economic burden of obesity and cardiometabolic disorders in the UAE, given the lack of such an assessment. We hope that our reported data provide an up-to-date epidemiological profile (prevalence, incidence, and trends) for the country with regard to cardiometabolic disorders and their associated costs and that this would eventually guide public health policy-makers in prioritizing and allocating resources properly for managing and preventing those diseases [9]. We also hope that our review highlights the current gaps in relevant research in preparation for subsequent research efforts.

\section{Methods}

We followed the standardized methods outlined by Moher et al. in the PRISMA 2010 group in conducting and reporting our systematic review [10]. However, we drafted a priori a protocol for the review and published it in the International Prospective Register of Systematic Reviews (PROSPERO) (CRD42016035747).

2.1. Database Search. We searched MEDLINE, PubMed, Embase, Cumulative Index to Nursing and Allied Health Literature (CINAHL), Index Medicus for the Eastern Mediterranean Region (IMEMR), ProQuest Dissertations \& Theses Database (PQDT), Open Access Theses and Dissertations (OATD), and Web of Science for studies addressing the epidemiology and costs of cardiometabolic disorders in the UAE, using an extensive and highlysensitive search strategy (Appendix A-a in Supplementary Materials). We replicated the search employing appropriate and specifically adapted vocabulary for each of the databases searched in order to retrieve all potentially eligible references. The initial search was conducted on June 19, 2015, and updated on April 4, 2017, to ensure that our review is current.

2.2. Searching Other Sources. In addition to the above databases, we searched the grey literature, the International Diabetes Federation (IDF), and primarily the World Bank websites for additional eligible studies. We also contacted prominent scholars and experts in the field from the UAE to inquire about any ongoing relevant studies not published yet.

It is worth mentioning that, in our original search, we aimed at finding studies that address the epidemiology and costs of either cancer and/or cardiometabolic disorders in the UAE, which are the top two incident NCDs within the UAE and thus the lead priorities on its public health agenda [6]. However, given the difference between the two diseases with regard to their underlying determinants (i.e., risk factors) and patient profiles and in order to generate diseasespecific epidemiology and/or cost data for specialized policymakers and researchers in each field, we opted to report the data for each of them in a separate systematic review. Nonetheless, both reviews were registered with the same protocol (CRD42016035747).

2.3. Inclusion and Exclusion Criteria. As outlined in our protocol, studies eligible for inclusion had to meet the following criteria:

(i) Be original studies (e.g., editorials, case reports, case series, and reviews were excluded)

(ii) Have an observational design: cohort or crosssectional studies for epidemiological outcomes and economic models and cross-sectional or longitudinal studies for cost analyses

(iii) Address cardiometabolic disorders defined as cardiovascular diseases of all types, type 2 diabetes, and/or metabolic syndrome including its primary components of obesity, insulin resistance, dyslipidemia, and/or hypertension

(iv) Report data specific for the UAE's citizens irrespective of their nationality (nationals and/or expatriates), sex (women and/or men), or age (children and/or adults)

(v) Written in English, Arabic, or French, irrespective of publication status (published versus unpublished) or date (i.e., no time limit)

2.4. Selection of Studies. Three reviewers (RB, HR, and RR), assisted by a reference manager (EndNote $\odot$ ), looked for potentially eligible studies by screening the titles and abstracts of the records retrieved by the search. After conducting a prior calibration exercise to ensure inter-reviewer screening consistency, two pairs of authors (RB/RR and $\mathrm{HR} / \mathrm{HH}$ ) individually and in pairs retrieved and evaluated the full texts of one-half of the references deemed eligible before (i.e., during the title and abstract screening phase) for inclusion in data extraction. A screening tool was developed 
by the three reviewers ( $R B, H R$, and $R R$ ) and pretested through a calibration exercise prior to the actual full-text screening. Disagreements were resolved through discussion with a third reviewer.

2.5. Data Extraction. The two reviewers in each pair $(\mathrm{RB} / \mathrm{RR}$ and $\mathrm{HR} / \mathrm{HH}$ ) individually and in pairs extracted relevant data from the included studies, consulting a third reviewer whenever they disagreed. As set in our protocol, the reviewers performed a qualitative (i.e., narrative) synthesis of the data extracted from the included studies, given that quantitative synthesis (i.e., meta-analysis) is not possible due to the epidemiological nature of the data extracted.

2.6. Risk of Bias Assessment. The reviewers used the tool developed and validated by Hoy et al. [11] to assess the risk of bias in the included studies. The tool comprises 10 items that address both the external and internal validity of each study, with an additional item that provides an overall summary of the risk of bias in the study. Each item is categorized as having "high risk," "intermediate risk," or "low risk" of bias, with the overall risk of bias being lower when more criteria (i.e., items) are adequately met. A high risk of bias was assigned to studies with unclear or poor reporting of a particular item. Finally, a study was considered to have an overall high risk of bias when it met less than 5 criteria, moderate risk of bias when it met 5 to 7 criteria, and low risk of bias when it met 8 or more criteria.

\section{Results}

Our initial search retrieved 6789 records, of which only 30 were deemed eligible. The updated rerun of the search retrieved 6 additional eligible studies, yielding a total of 36 studies that were eligible and included in data extraction and synthesis (Appendix A-b in Supplementary Materials).

3.1. Characteristics of Included Studies. Tables 1-3 summarize the characteristics, results, and overall risk of bias in the included epidemiological studies $(n=35)$. Table 4 summarizes the characteristics and results of the single study identified that addresses the cost of illness (COI) for diabetes [12]. Included studies addressed various cardiometabolic disorders, including overweight/obesity $(n=19)$, metabolic syndrome $(n=3)$, dyslipidemia $(n=1)$, hypertension $(n=2)$, diabetes $(n=4 ; 2$ prevalence, 1 incidence, and 1 cost), and multiple cardiometabolic disorders at once $(n=7)$. Only one study was a retrospective cohort [13], with the rest being cross-sectional studies $(n=35)$. All included studies were published between 1995 and 2016 (1990-1999: $n=6$; 2000-2009: $n=15$; and after 2009: $n=15$ ), reporting data collected between 1989 and 2015. Half of the included studies reported data specific to Emiratis $(n=18)$, and twothirds $(n=24)$ reported data for both sexes. Only six studies were conducted at the national level $[3,14-18]$, with the rest being emirate-specific. Finally, more than half of the included studies used a random sample $(n=20)$, seven recruited a convenient sample, four adopted exhaustive sampling (census), and one included a purposive sample. Four studies did not report their sampling method. It is worth noting that the included studies remarkably varied in their recruited sample size, ranging from 98 [19] up to 173,501 [20] participants.

\section{The Epidemiology of Cardiometabolic Disorders in the UAE}

\subsection{Overweight and Obesity}

4.1.1. Paediatric Population. Abdulrazzaq et al. (1991-1992) provided the earliest and most exhaustive national-level estimates of the prevalence of overweight and obesity in the Emirati paediatric population, reporting rates of $13 \%$ for boys below the age of 5 and $6 \%$ for their female counterparts [16]. The study reports that these figures gradually increase with age, peaking at 18 years. Three other studies address more recent national estimates for overweight and obesity in Emirati schoolchildren and adolescents, with a 10-year interval difference between the two older studies (1998-1999) [22] and the most recent one (2009-2010) [3]. Despite reporting similar overweight estimates of about $19 \%$ for each of the boys and the girls and no major differences in sexspecific rates, the two older studies $[15,22]$ remarkably differed in their reported overall obesity rates (7\% [22] versus $13 \%[15])$. In contrast, the more recent study highlights the emergence of sex-specific differences, particularly in overweight rates in children aged 6 to 10 (24\% for girls versus $9 \%$ for boys) and obesity rates in adolescents aged 11 to 18 (20\% for girls versus $12 \%$ for boys) [3]. The study reports an overall prevalence of $40 \%$ of overweight and obesity for Emirati female children compared to $25 \%$ only for their male counterparts [3]. The same was reported in adolescents. On the contrary, at the emirate-specific level, Fujairah seems to harbour the highest rates of overweight and obesity, reporting a striking 30\% prevalence among its Emirati schoolgirls [21]. Two other studies assessed the prevalence of overweight and obesity in Emirati schoolchildren in Ras $\mathrm{Al}$ Khaimah, another emirate, with more than a decade as the time difference between the older study [14] and the recent one [23]. Direct comparison of the results of these two studies $[14,26]$ is challenging given their use of different definitions for overweight and obesity and the inclusion of children of different age groups. However, despite those differences, the rates of overweight and obesity reported by the older study compared to the recent one show a tremendous increase over time, almost doubling for overweight (9\% versus $17 \%$, respectively) and more than doubling for obesity ( $8 \%$ versus $20 \%$, respectively). As for Abu Dhabi, two recent studies report similar rates of approximately $34 \%$ of overweight and obesity prevalence among both Emirati and non-Emirati schoolchildren $[25,35]$. These studies have also employed different disease definitions and included children of different age groups, which prevented us from providing conclusive evidence through directly comparing their findings. However, the two studies seem to be in agreement with regard to their reported 


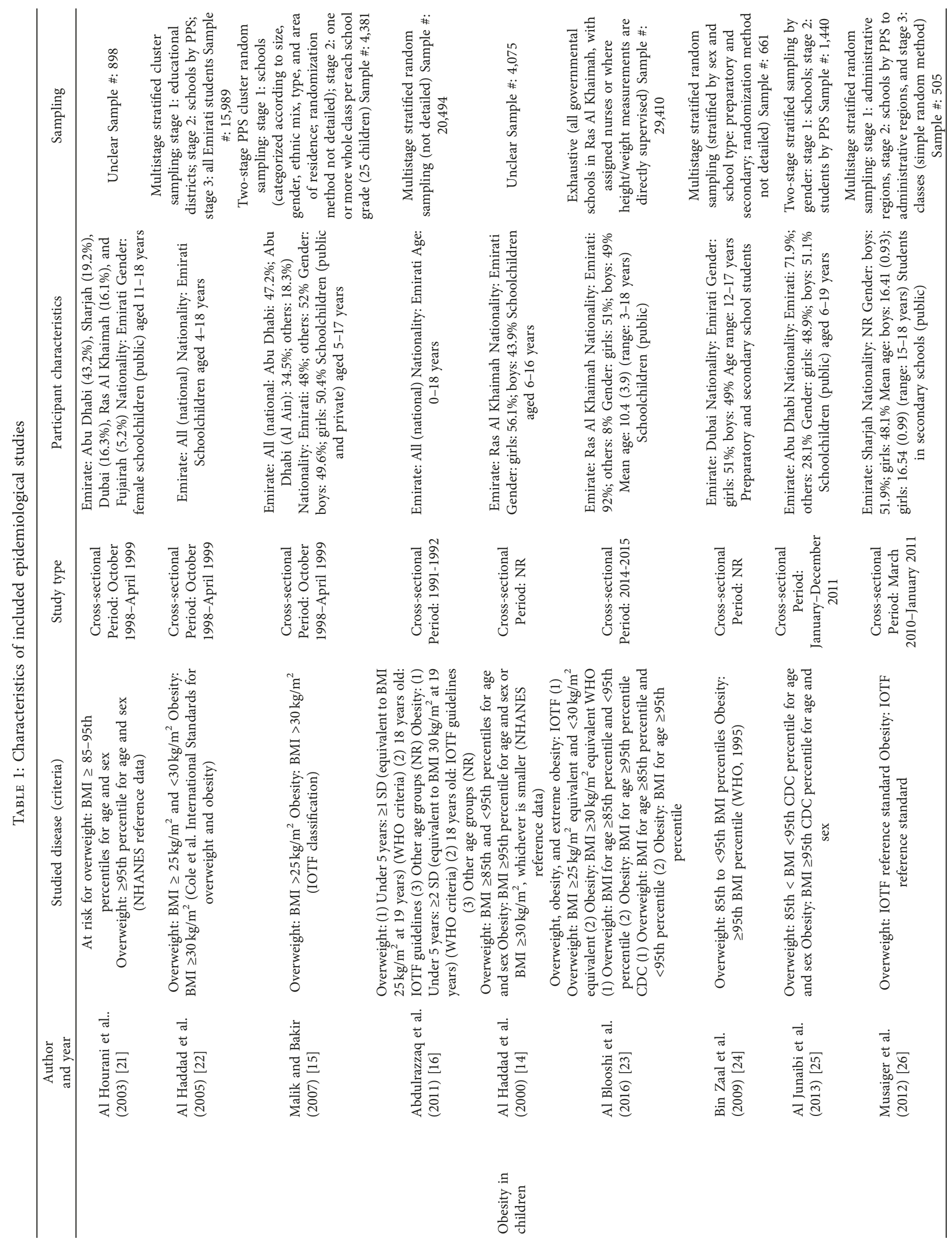




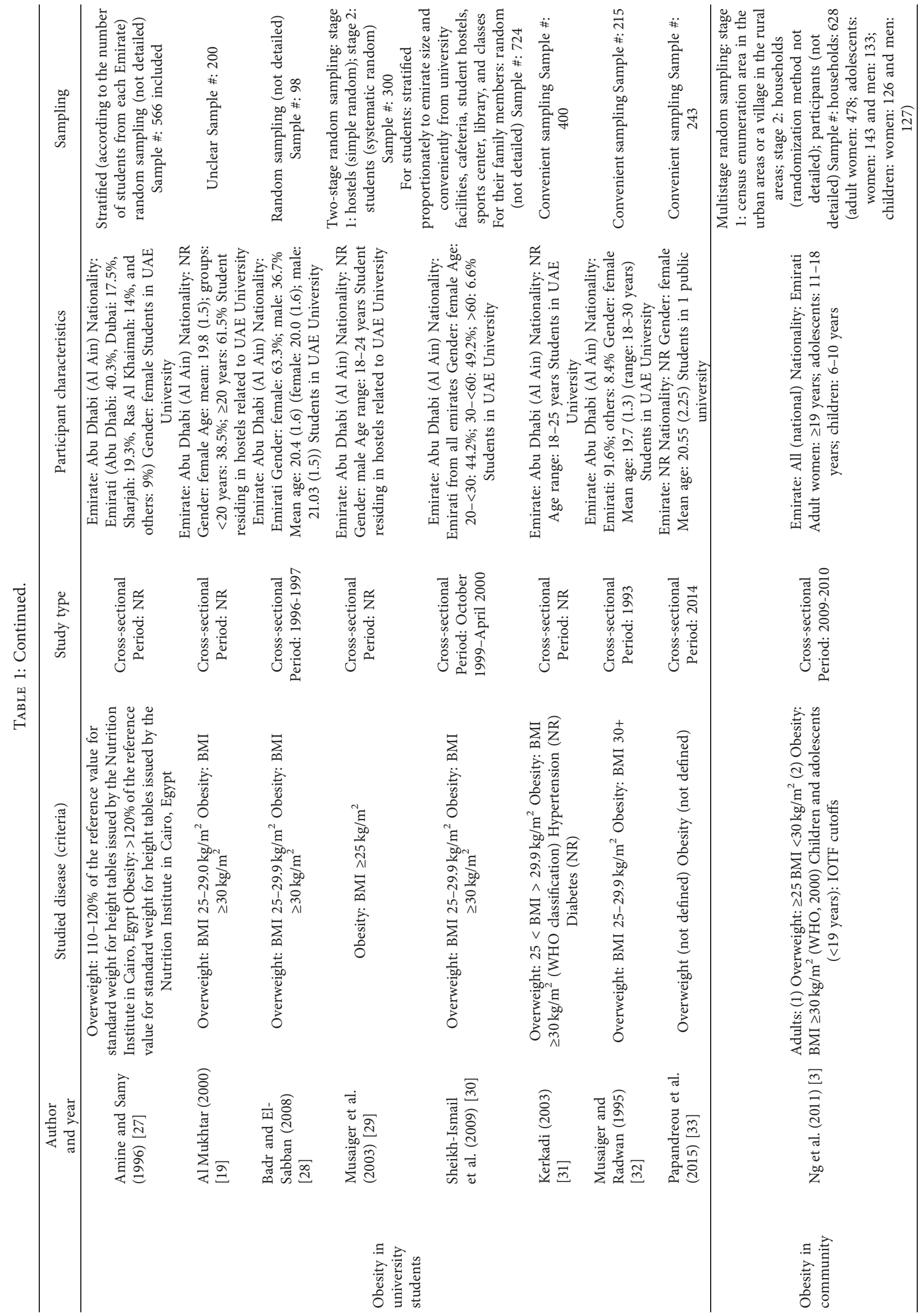




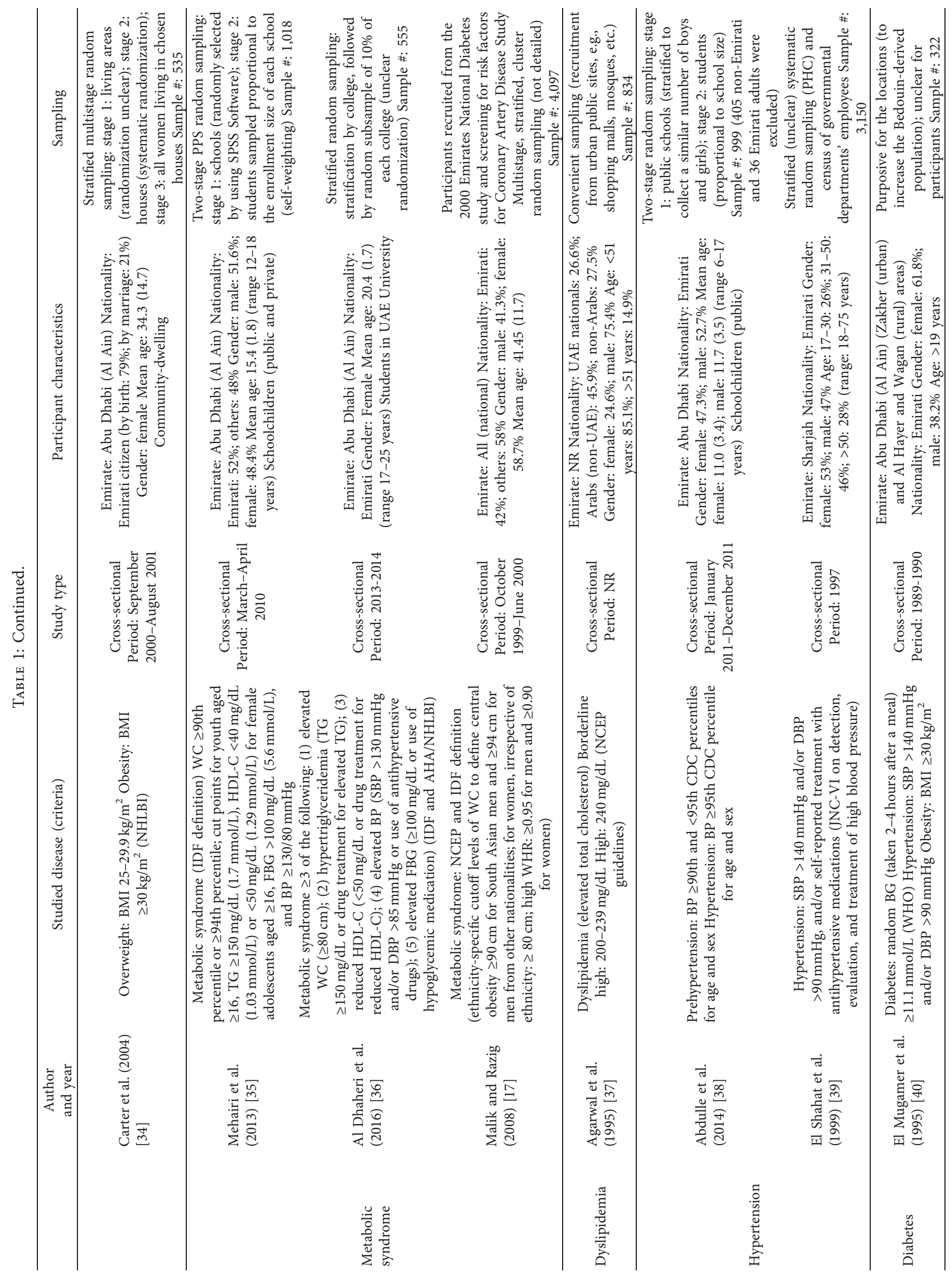




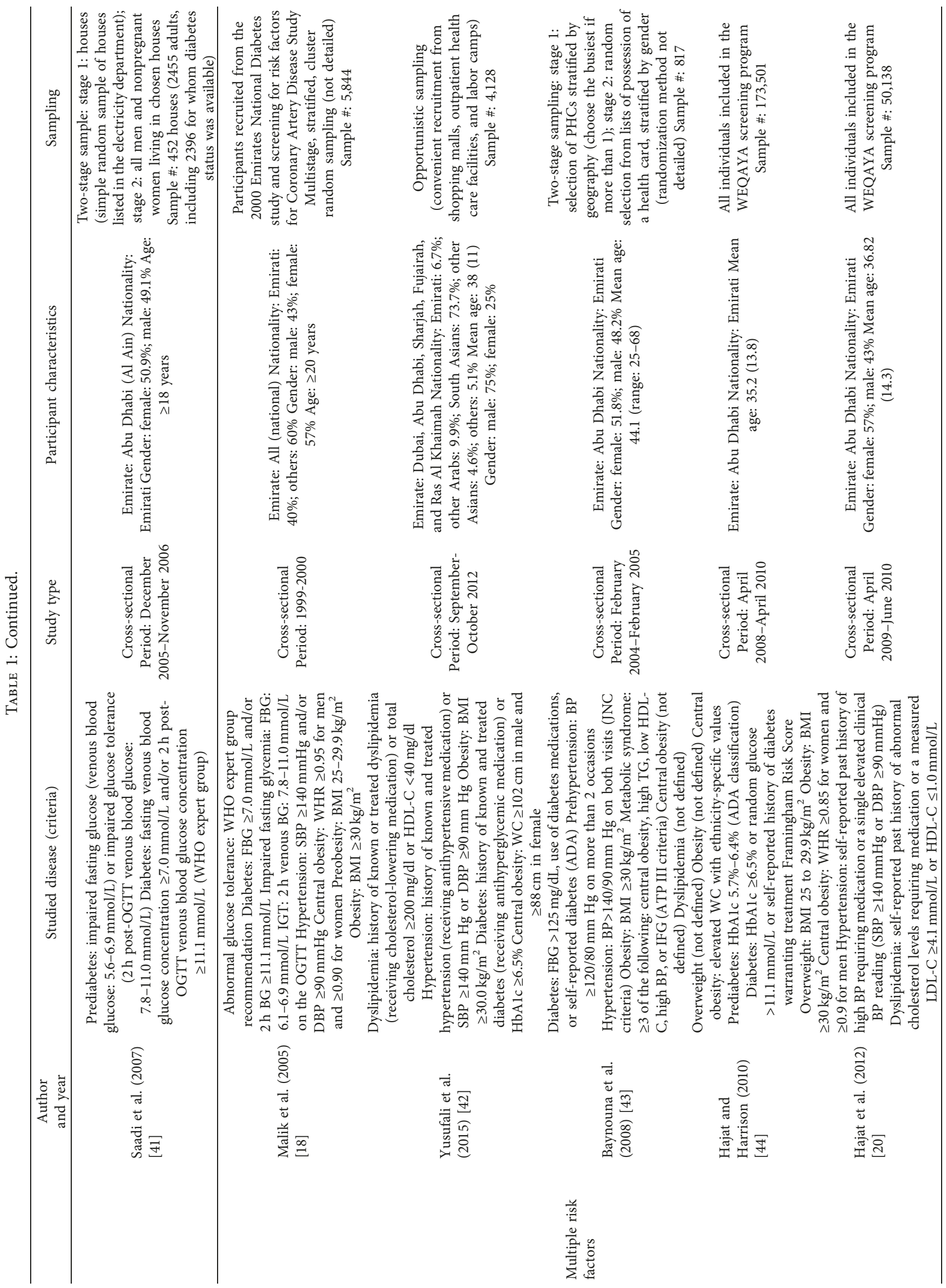




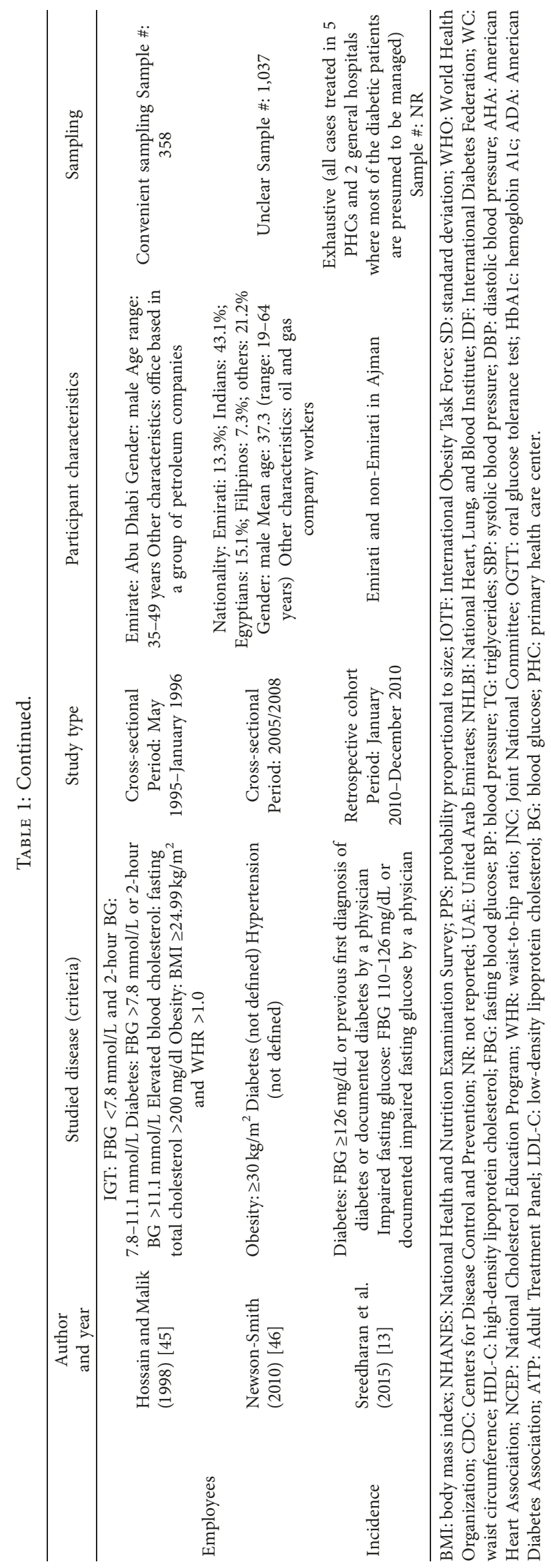




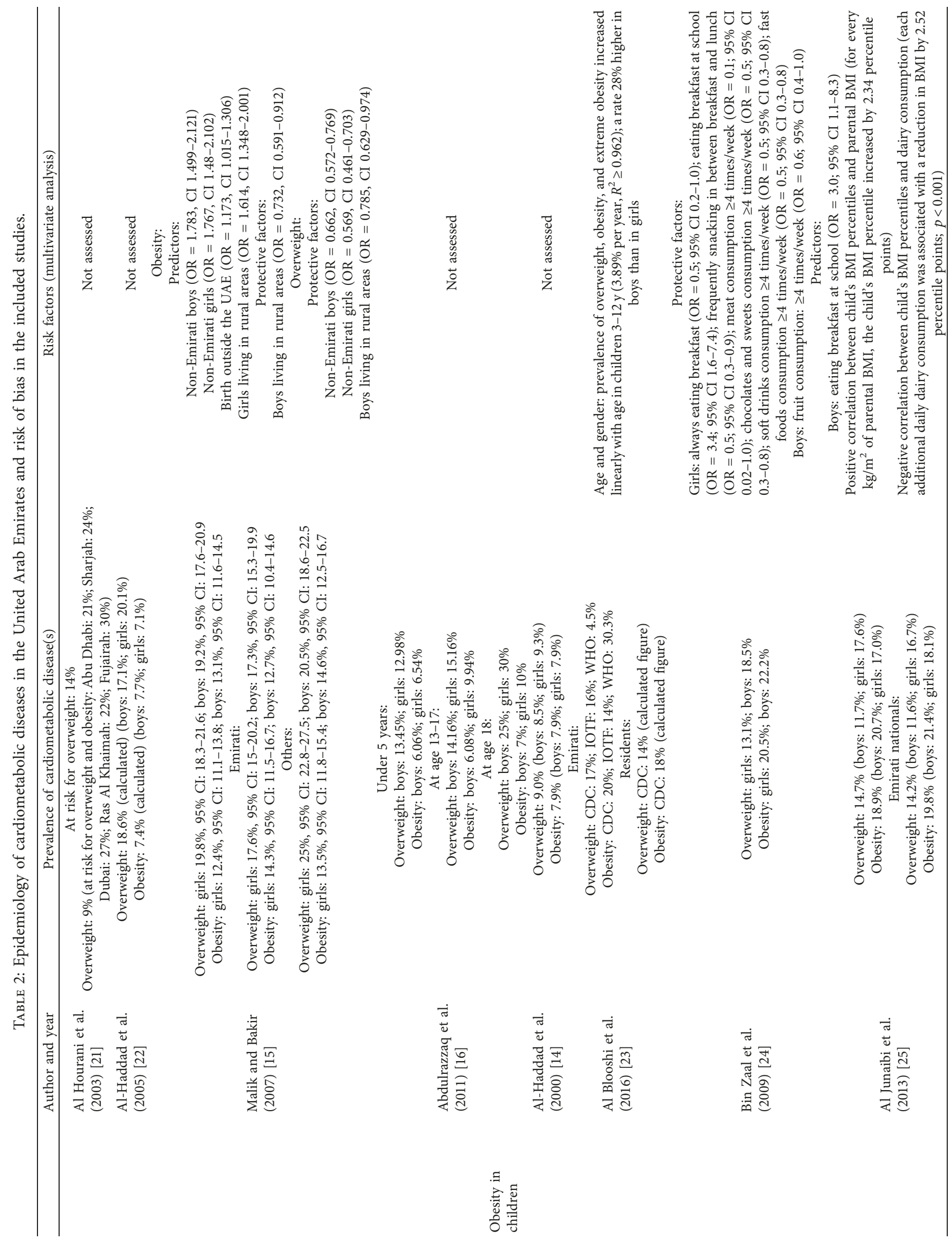




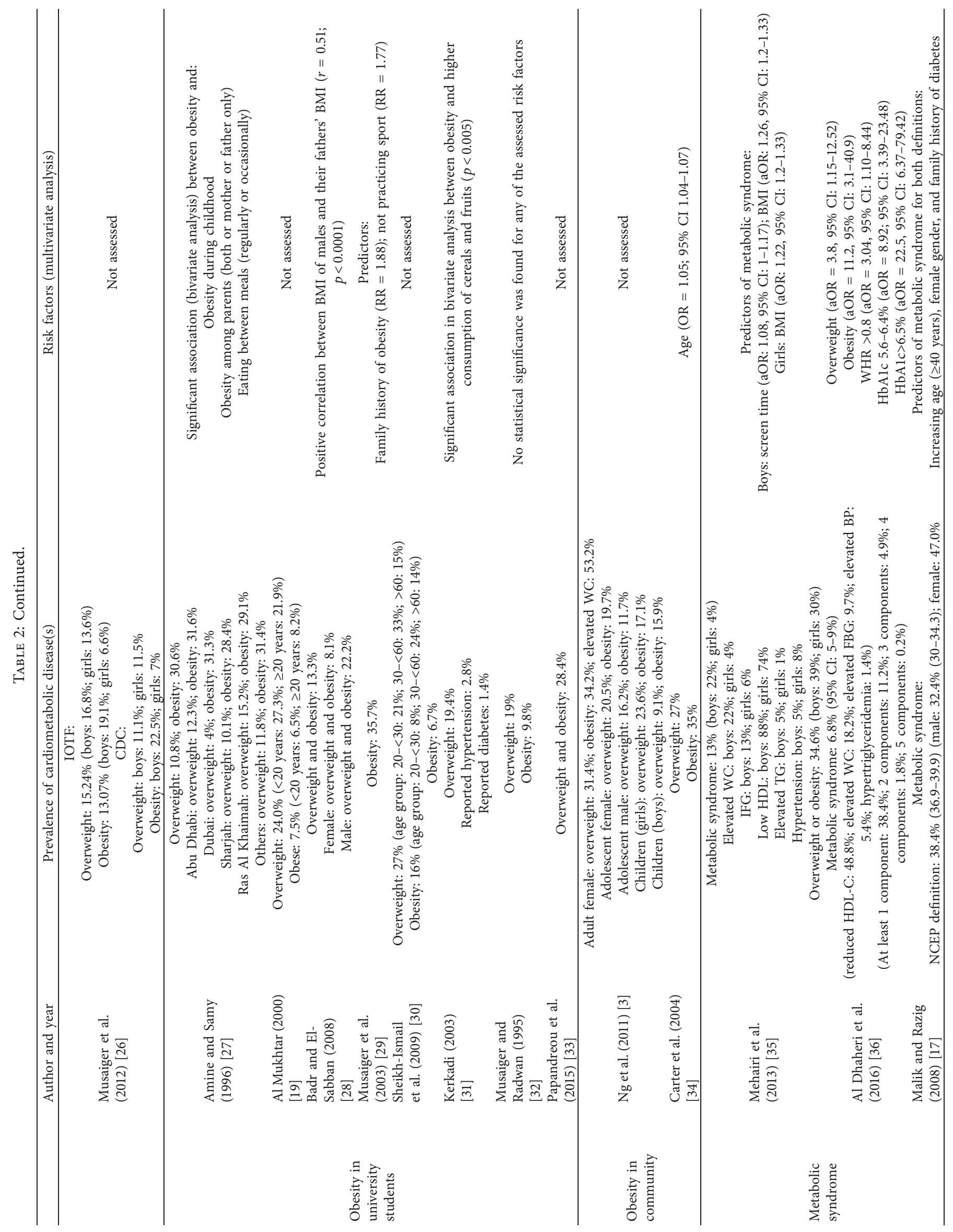




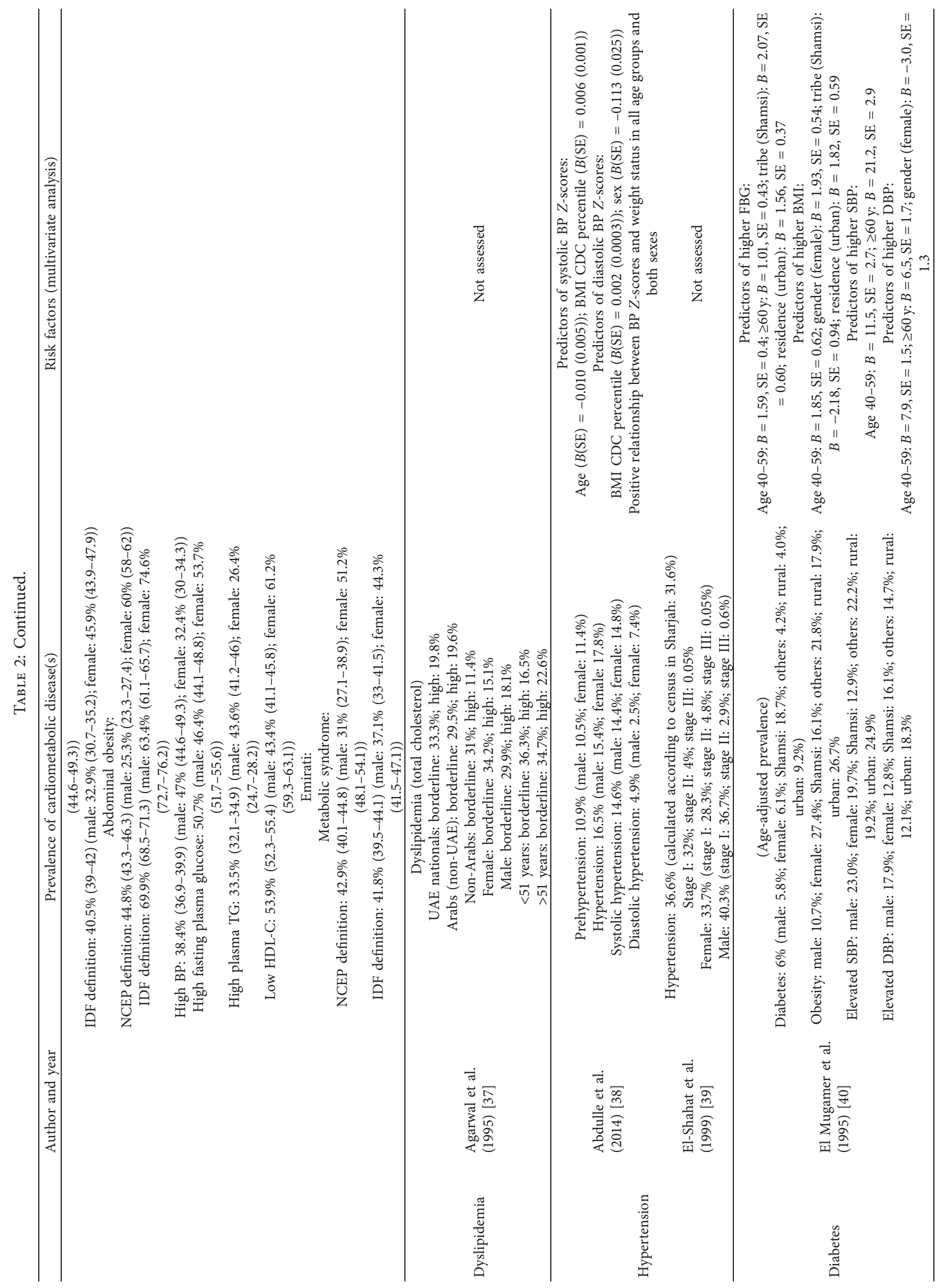



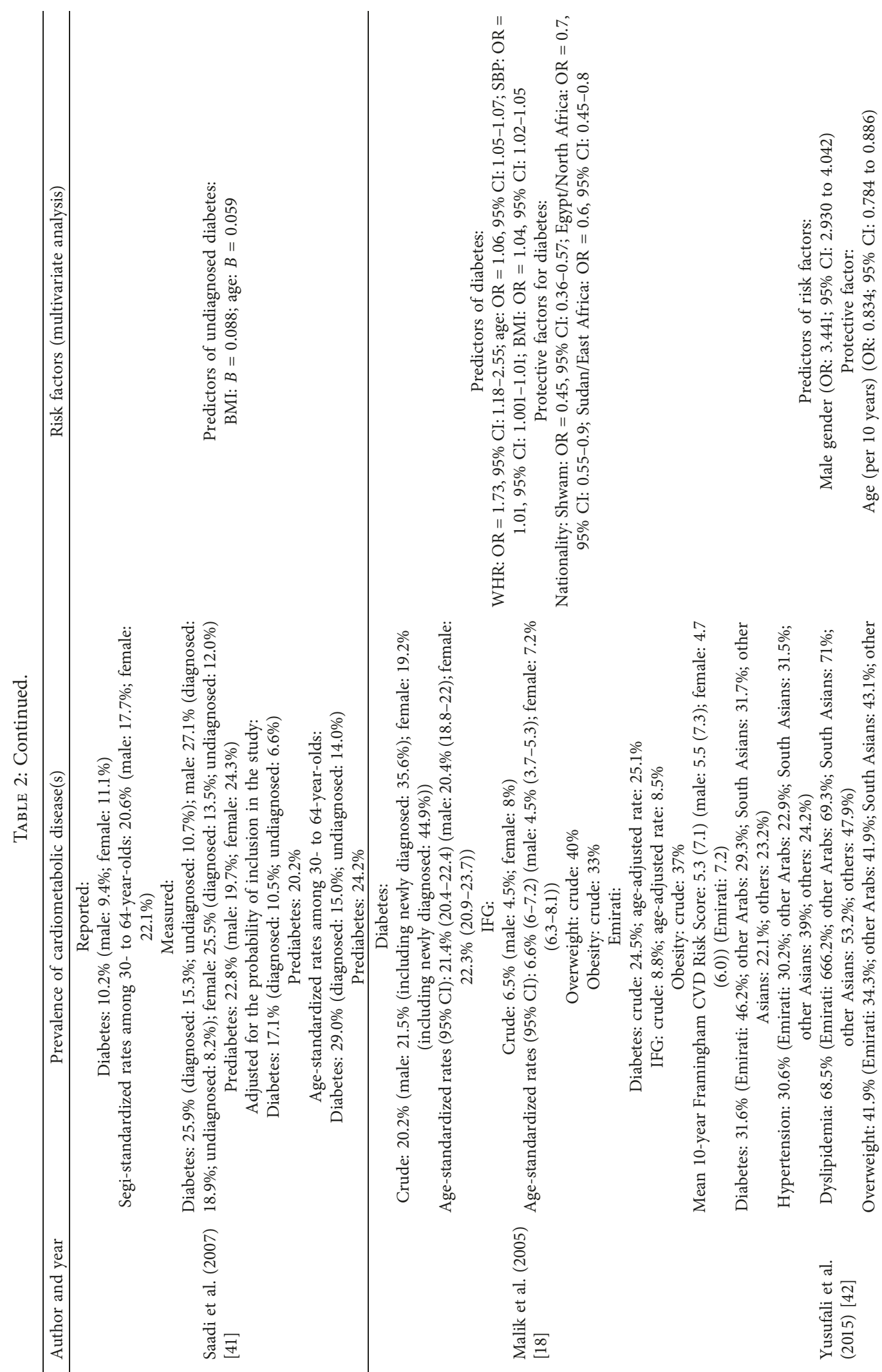

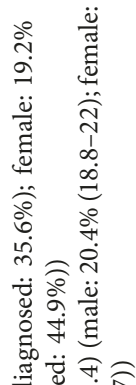

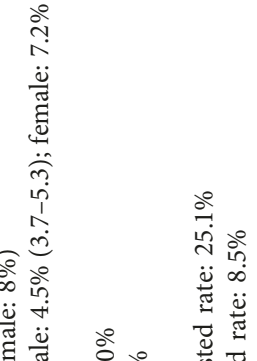

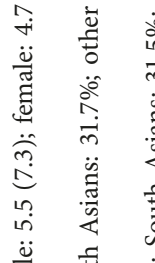

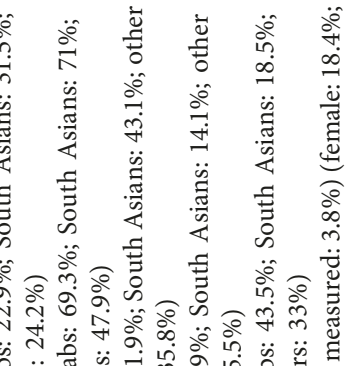

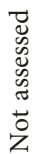

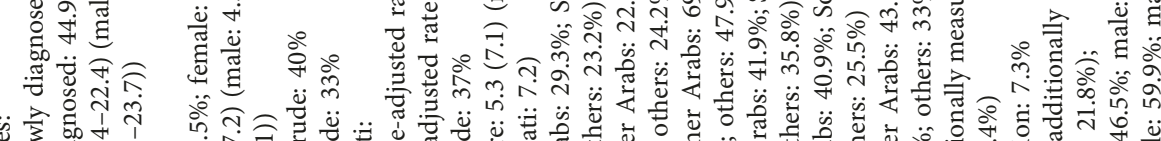

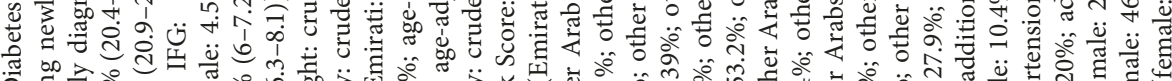

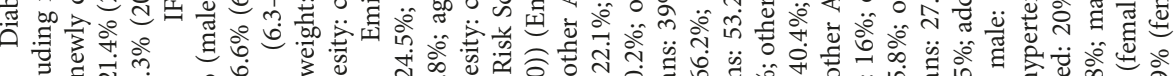
हु

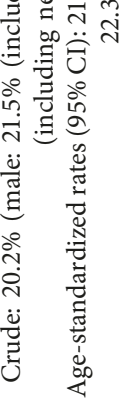

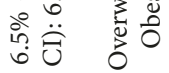

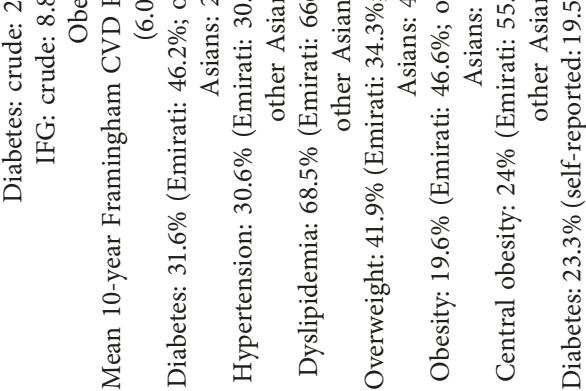
늘

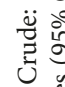

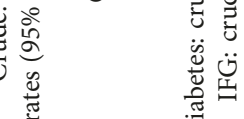

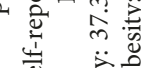
ㅇํำ

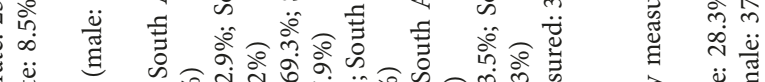




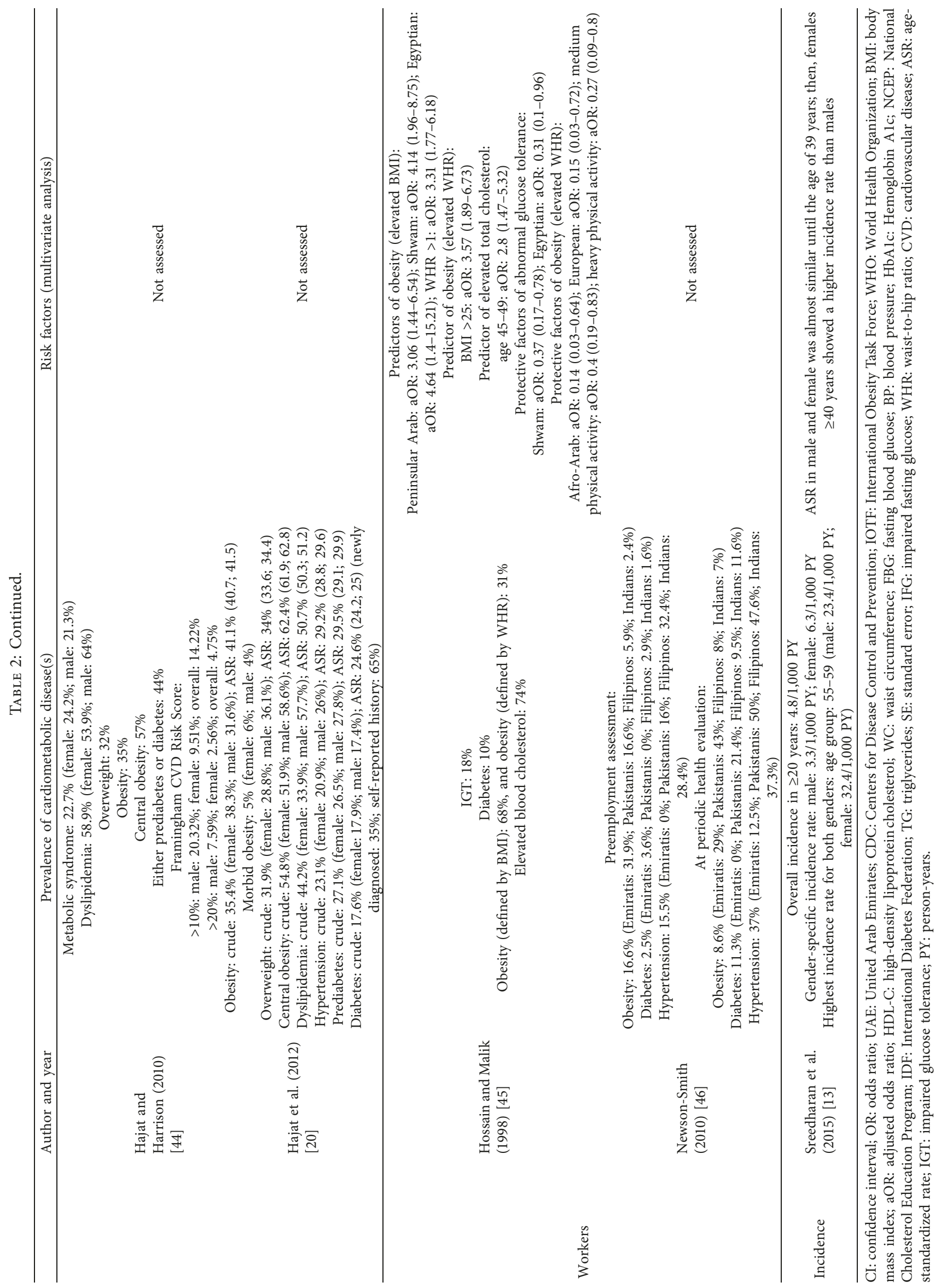




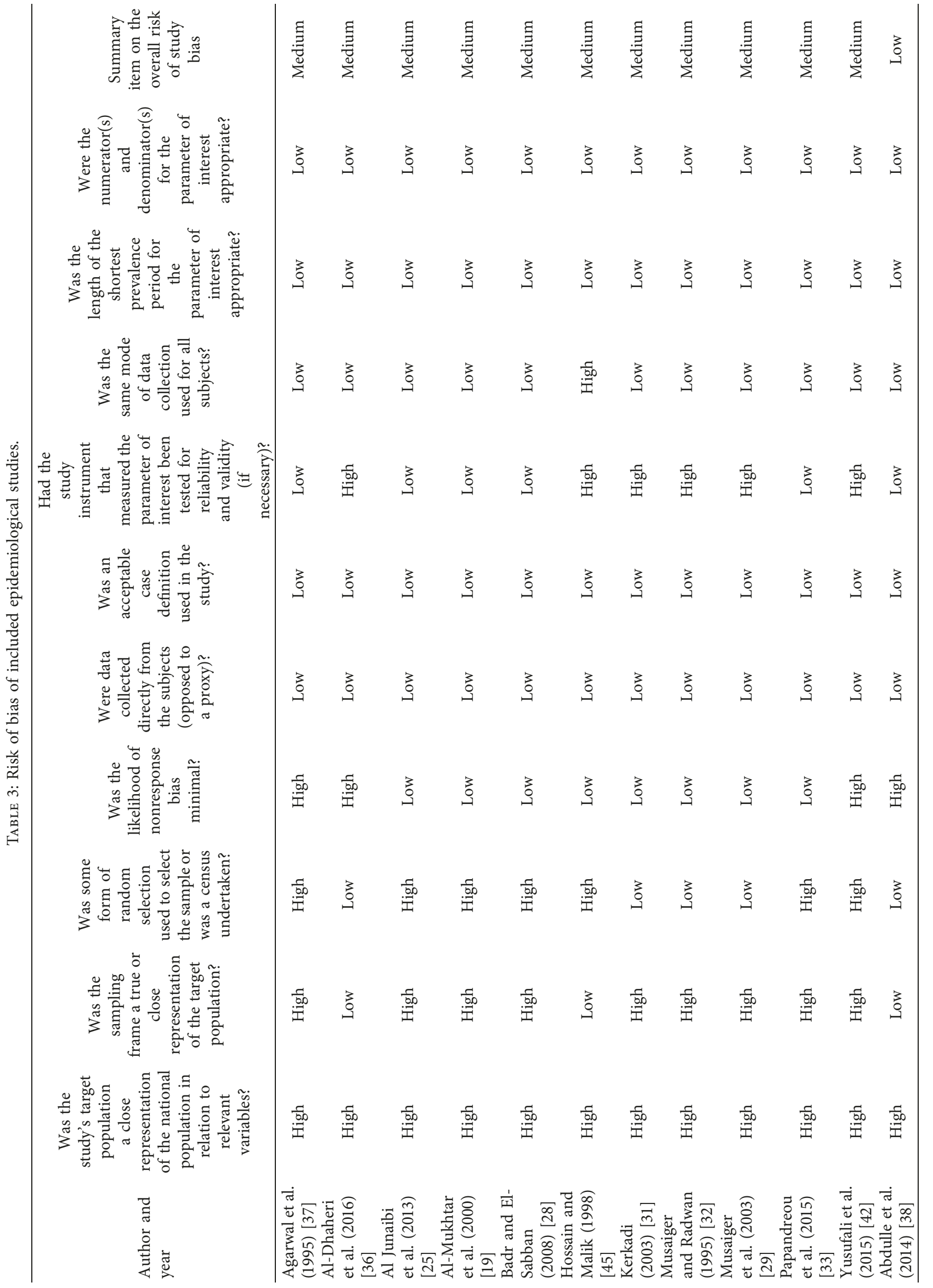




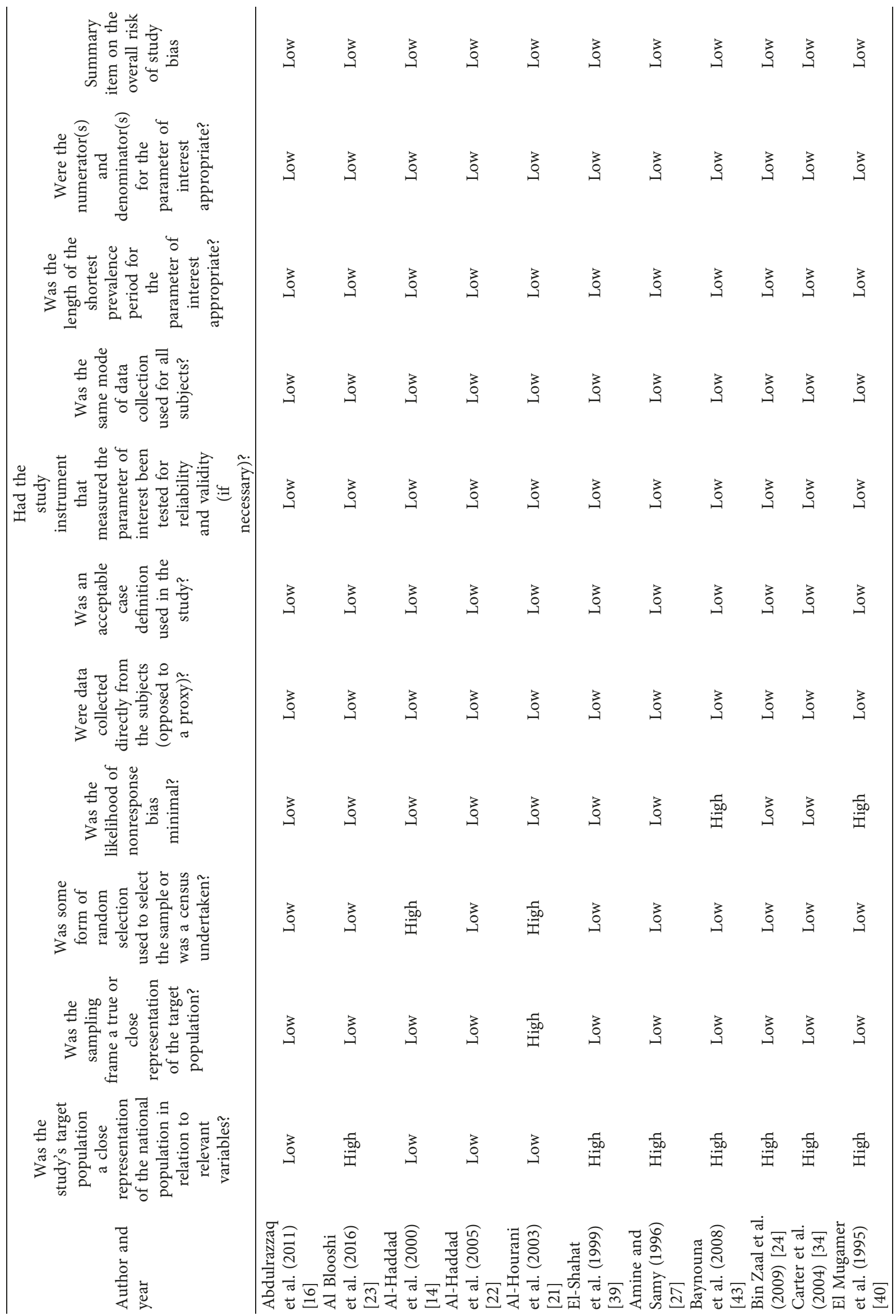




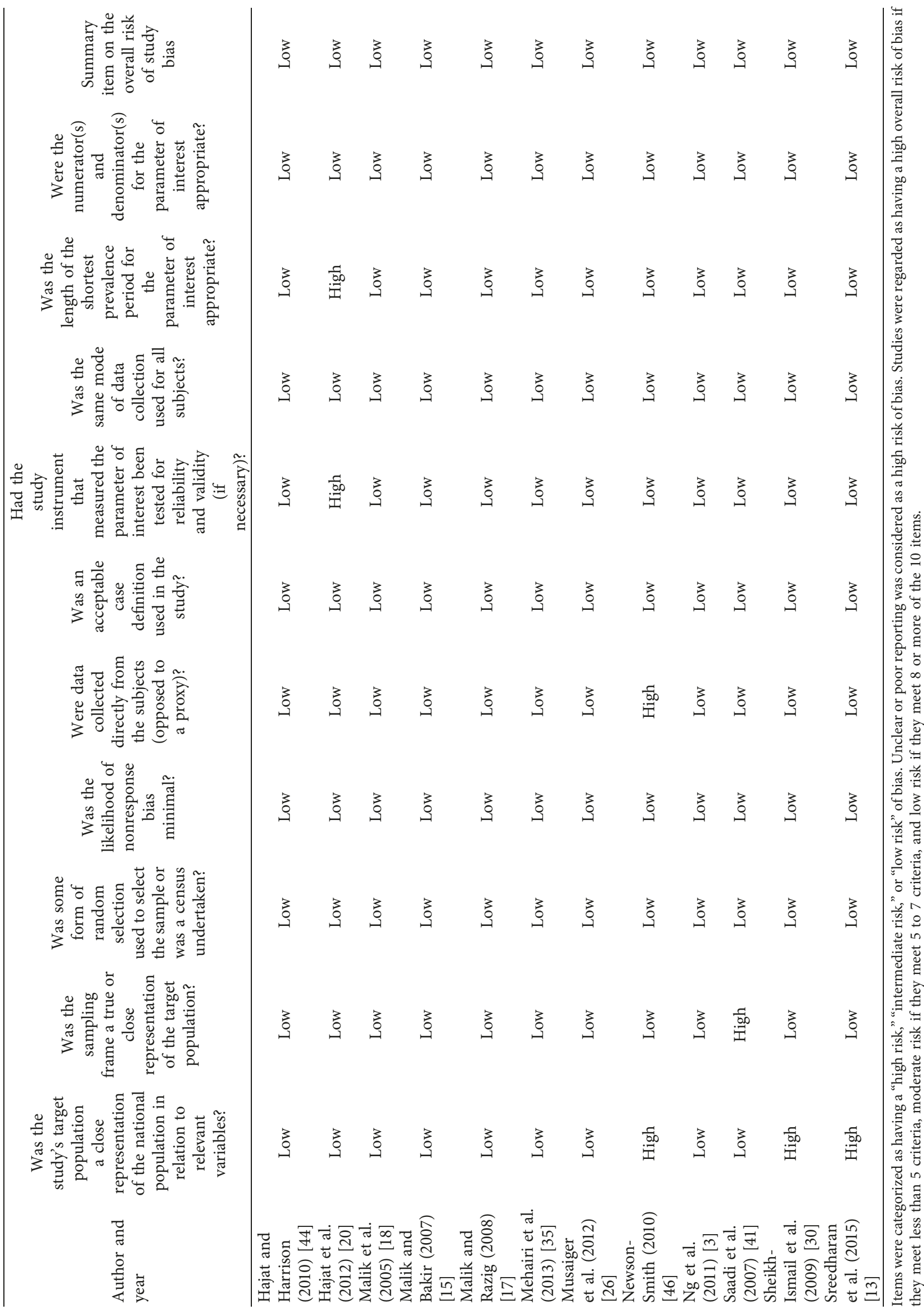


sex-specific rates, highlighting a greater proportion of overweight and obesity in boys compared to girls in $\mathrm{Abu}$ Dhabi. In contrast, Dubai shows almost equal rates of obesity in the two sexes (girls $21 \%$ and boys $22 \%$ ), yet higher rates of overweight in boys (19\%) compared to girls (13\%) [24]. Conversely, Sharjah shows identical overweight rates among its boys and girls $(11 \%)$, with more than triple the rate of obesity in its boys (23\%) compared to girls (7\%) [26].

4.1.2. University Students. All studies addressing the rates of cardiometabolic disorders among university students were conducted at the UAE University in Al Ain, Abu Dhabi. They report varying rates of overweight and obesity across female university students, ranging between 29\% [32] and $46 \%$ [30]. In contrast, only one study tackled male university students and reported an obesity rate of 35\% among them [29] without reporting their overweight rates.

4.1.3. Adults. A study conducted at the national level between 1999 and 2000 reported prevalence rates of $40 \%$ and $30 \%$ for overweight and obesity, respectively, in Emirati and non-Emirati adults combined [14]. In contrast, a more recent study reports a prevalence of $42 \%$ for overweight and $20 \%$ for obesity in 2012 among the same abovementioned population [42]. However, the latter study recruited a convenient sample of Emirati and non-Emirati adults in 5 of the UAE's 7 emirates, making the deduction of temporal trends somewhat unreliable. Regarding sex-specific rates in the adult population, a recent national study shows that nearly two-thirds of adult Emirati females carry excess body weight (31\% overweight and 34\% obese) [3], yet it does not report any estimates for males. However, an earlier study found remarkable differences in sex-specific rates of obesity, reporting a prevalence of $47 \%$ in women compared to $28 \%$ in men [43]. The same study reported an overall estimate of $37 \%$ for overweight and obesity in Emirati adults residing in Abu Dhabi [43]. Likewise, in a study reporting emiratespecific rates, it was estimated that around three-quarters of the Emirati adults residing in Abu Dhabi carry excess body weight, with $34 \%$ and $41 \%$ of them being overweight and obese, respectively [20].

4.2. Metabolic Syndrome. The national estimates of metabolic syndrome among Emirati and non-Emirati adults were $38 \%$ and $41 \%$, respectively, in 1999-2000, using the National Cholesterol Education Adult Panel III (NCEP) and IDF definitions [17]. Interestingly, regardless of the definition used, the rate of metabolic syndrome appears to be higher in females compared to males ( $47 \%$ and $46 \%$ versus $32 \%$ and $33 \%$, according to the NCEP and IDF definitions, respectively). Particularly, the two components that were more accentuated in females compared to males in the NCEP definition were a low level of high-density lipoprotein cholesterol (HDL-C) (54\% of cases) and a high fasting plasma glucose (51\% of cases) [17]. As for schoolchildren in Abu Dhabi, around 13\% of those aged 12 to 18 years suffer from metabolic syndrome as defined by the IDF, although with a greater prevalence in boys (22\%) than girls (4\%) [35]. According to that study, the most common components of the IDF definition that remarkably differed between the two sexes were a low HDL-C and an elevated waist circumference. Finally, among university students, only $7 \%$ of the female Emirati students attending the UAE University were found to have metabolic syndrome [36], with $38 \%$ of the study's participants satisfying at least one component of the IDF definition of metabolic syndrome [36].

4.3. Dyslipidemia. One study particularly addressing dyslipidemia, defined as a total cholesterol $>240 \mathrm{mg} / \mathrm{dL}$, reports prevalence estimates of $19 \%$ in Emirati and non-Emirati Arabs compared to $11 \%$ in non-Arabs [29]. The study also shows increasing rates with advancing age $(23 \%$ in those $>51$ years old versus $17 \%$ in younger participants). A more recent study addressing dyslipidemia estimates in a convenient sample of adults from 5 emirates (Dubai, Abu Dhabi, Sharjah, Fujairah, and Ras Al Khaimah) reports a $69 \%$ overall rate of elevated total cholesterol $(\geq 200 \mathrm{mg} / \mathrm{dl})$ or reduced HDL-C level $(<40 \mathrm{mg} / \mathrm{dl})$ [42]. The study was conducted as part of assessing the cardiometabolic status of those emirates [42]. Two other studies conducted 5 years apart (2004-2005 [43] and 2009-2010 [20]) report comparable rates of dyslipidemia among the Emirati citizens of Abu Dhabi (59\% and 51\%, respectively), despite using different disease definitions. Interestingly, Hajat et al. highlight sex-based differences in the rates of dyslipidemia (defined as $\mathrm{LDL}-\mathrm{C} \geq 4.1 \mathrm{mmol} / \mathrm{L}$ or HDL-C $\leq 1.0 \mathrm{mmol} / \mathrm{L}$ ), reporting a prevalence of $57.7 \%$ in men compared to $33.9 \%$ in women [20].

4.4. Impaired Fasting Glucose, Prediabetes, and Diabetes. The sole study conducted at the national level in the UAE was between 1999 and 2000, reporting a prevalence of $21 \%$ for diabetes and 7\% for impaired fasting glucose (IFG) [18]. A decade later, another study addressing the prevalence of diabetes in a convenient sample from five emirates reported an increase in prevalence to $32 \%$ [42]. However, Abu Dhabi remains the only emirate in which diabetes prevalence can be trended, given the multiple studies on the topic since 1989 $[20,40,41,43]$. Of these studies, the oldest reports an agestandardized rate (ASR) of $6 \%$ for diabetes within a purposive sample of Bedouin Emirati adults between 1989 and 1990 [40]. The subsequent study, conducted more than a decade later, reports an increase in the ASR of diabetes to $17 \%$, with a concomitantly high ASR for prediabetes (20\%) [41]. Another study conducted afterward reports an even higher estimate for diabetes (23\%) [43]. Finally, the most recent of those studies states that more than half of the Emirati adults of Abu Dhabi suffer from dysglycemia, reporting increased estimates of both diabetes $(24.6 \%)$ and prediabetes $(29.5 \%)$, with no significant differences across the corresponding sex-specific rates [20]. Thus, by comparing the diabetes estimates reported in those studies in their respective chronological order, we clearly recognize an uptrend in the prevalence of diabetes in Abu Dhabi throughout the years. On the contrary, only one study addresses the incidence of diabetes in Ajman, reporting an 
TABle 4: Cost of illness for diabetes from Al-Maskari et al. [12].

\begin{tabular}{|c|c|c|c|c|c|c|c|}
\hline $\begin{array}{l}\text { Study } \\
\text { design }\end{array}$ & Sample size & Data sources & $\begin{array}{l}\text { Cost components } \\
\text { and costing } \\
\text { approach }\end{array}$ & Perspective & $\begin{array}{c}\text { Time } \\
\text { horizon }\end{array}$ & $\begin{array}{l}\text { Economic burden } \\
\text { (annual } \\
\text { cost/patient) (US\$, } \\
\text { 2004) }\end{array}$ & Major limitations \\
\hline $\begin{array}{l}\text { Cross- } \\
\text { sectional }\end{array}$ & $\begin{array}{l}150 \text { (recruited from } \\
2 \text { outpatient clinics } \\
\text { at Al Ain, Abu } \\
\text { Dhabi: } 67 \% \text { men; } \\
48 \% \text { nationals; } \\
33 \%:>60 \text { years old) }\end{array}$ & $\begin{array}{l}\text { Cost data: official } \\
\text { list of charges/rates } \\
\text { for patients not } \\
\text { covered by health } \\
\text { insurance } \\
\text { Resources use: } \\
\text { interviewer- } \\
\text { administered } \\
\text { questionnaire } \\
\text { completed by } \\
\text { patients }\end{array}$ & $\begin{array}{l}\text { Components: } \\
\text { direct costs (visits } \\
\text { to primary health } \\
\text { care centers or } \\
\text { diabetes clinic, } \\
\text { laboratory tests, } \\
\text { medications, } \\
\text { hospitalizations } \\
\text { due to diabetes } \\
\text { complications, and } \\
\text { emergency room } \\
\text { visits due to } \\
\text { diabetes) } \\
\text { Approach: marco- } \\
\text { costing }\end{array}$ & $\begin{array}{l}\text { Health } \\
\text { care payer }\end{array}$ & 1 year & $\begin{array}{c}\text { No complications: } \\
\text { US } \$ 1,605 \text { (\$2,015 } \\
\text { adjusted to 2015) } \\
\text { Microvascular } \\
\text { complications: } \\
\text { US } \$ 3,453 \text { ( } \$ 4,334 \\
\text { adjusted to 2015) } \\
\text { Macrovascular } \\
\text { complications: } \\
\text { US } \$ 10,300 \\
\text { (\$12,929 adjusted } \\
\text { to 2015) } \\
\text { Micro- and } \\
\text { macrovascular } \\
\text { complications: } \\
\text { US } \$ 15,104 \\
\text { (\$18,959 adjusted } \\
\text { to 2015) } \\
\text { Cost drivers: } \\
\text { diabetes-related } \\
\text { complications; } \\
\text { treatment with } \\
\text { insulin }\end{array}$ & $\begin{array}{l}\text { Self-reported } \\
\text { questionnaire, } \\
\text { uncertainties not } \\
\text { thoroughly } \\
\text { addressed, and } \\
\text { sensitivity analyses } \\
\text { not conducted }\end{array}$ \\
\hline
\end{tabular}

overall rate of 4.8 per 1,000 person-years (PY), with its highest rates being seen in those aged 55 to 59 years $(23.4$ and 32.4 per 1,000 PY for men and women, respectively) [13]. That study also shows that the incidence rates of diabetes among women remarkably increase after the age of 40 , becoming almost double the corresponding rates for men (6.3 versus 3.3 per 1,000 PY, respectively).

4.5. Prehypertension and Hypertension. One study addressed the prevalence of hypertension in 5 emirates and reported an overall estimate of $31 \%$ for hypertension prevalence in adults [42]. Hypertension was defined as having a history of known and treated hypertension, having a systolic blood pressure equal to or above $140 \mathrm{~mm} \cdot \mathrm{Hg}$, or having diastolic blood pressure equal to or above $90 \mathrm{~mm} \cdot \mathrm{Hg}$ [42]. At an emiratespecific level, only one study addressed the prevalence of hypertension in the Emirati adults of Sharjah, reporting an ASR of 37\% [39], which is much higher than rates reported by the two studies addressing hypertension prevalence in Abu Dhabi's Emirati adults (21\% in the older study [40] and $29 \%$ in the more recent one [25]). However, despite the increase in the overall estimates of hypertension in $\mathrm{Abu}$ Dhabi reported by the two latter studies (from 21\% to 29\%), it is difficult to accurately state whether this increase truly reflects an uptrend in hypertension prevalence, given the differences in the methodologies adopted by the two studies and the definitions used for elevated blood pressure. Interestingly, however, a higher prevalence of hypertension is noted in males compared to females in all three aforementioned studies $[20,39,43]$. As for the paediatric population, only one study addressed the prevalence of hypertension and reported that more than one-quarter of the Emirati schoolchildren residing in Abu Dhabi have elevated blood pressure (11\% prehypertensive and 17\% hypertensive, with a predominance of systolic hypertension in the hypertensive children) [42].

4.6. Cardiometabolic Disorders in the Working Class. Only two of the included studies addressed the prevalence of cardiometabolic disorders in adult employees in particular $[45,46]$. The older study reported a prevalence of $74 \%$ for dyslipidemia and $68 \%$ for obesity in both national and expatriate male workers [45]. In contrast, the more recent study compared the rates of cardiometabolic disorders in oil and gas company male workers of Abu Dhabi prior to employment to rates seen at postemployment periodic health evaluations [46]. It demonstrated an increase in hypertension and diabetes rates by about $20 \%$ and $9 \%$, respectively, with a decline in obesity rates by almost one-half (from $16.6 \%$ to $8.6 \%$ from preemployment to postemployment), all occurring within 3 years of employment [46].

Assessment of the included studies revealed that onethird of them had a medium overall risk of bias (11/35), and the remaining ones had a low risk of bias (24/35). The most commonly encountered defects in the included studies were failing to recruit truly representative samples of the target population $(n=21)$, employing a nonrepresentative sampling frame $(n=11)$, and recruiting nonrandom (i.e., convenient) samples $(n=9)$. Table 3 summarizes the risk of bias assessment for the included studies. 
4.7. Cost of Illness (COI) for Diabetes. Only one of the included studies addressed the COI for diabetes, one of the cardiometabolic disorders of interest to us [12]. This crosssectional study, which was conducted in 2004, adopted a health care-payer perspective that accounted for all the direct medical costs of diabetes for Emirati and non-Emirati patients attending the outpatient clinics at the two major referral hospitals (i.e., $\mathrm{Al}$ Ain and Tawam) regardless of age or sex. Table 4 summarizes the characteristics and results of the study. In short, using a macro-cost approach, the study estimates the annual cost of diabetes to be around US $\$ 1,605$ for patients with complication-free cases $(\$ 2,015$ adjusted for the year 2015). In contrast, this value almost doubles for patients with microvascular complications, increases by more than sixfold for those with macrovascular complications, and increases by more than ninefold for those with concomitant micro- and macrovascular complications [12]. However, the study's limited reporting of cost estimates, its failure to identify major uncertainties or perform any sensitivity analyses, and its insufficient documentation and justification for its reported estimates render its overall methodological quality of the suboptimal level (Appendix B in Supplementary Materials).

\section{Discussion}

This systematic review provides an overview of the prevalence of major cardiometabolic disorders in the UAE, namely, overweight and obesity, metabolic syndrome, dyslipidemia, diabetes, and hypertension.

The last study conducted to date at the national level in the UAE addressing overweight and obesity prevalence in Emirati and non-Emirati adults reported that the overall prevalence rates for overweight and obesity are $40 \%$ and $33 \%$, respectively, showing that almost three-quarters of the UAE's adults (73\%) have a body mass index (BMI) $\geq 25 \mathrm{~kg} / \mathrm{m}^{2}$ [47]. This somewhat mirrors the combined overall rates for overweight and obesity seen in the United States during the same period (64.5\%) [42]. However, a more recent study by Yusufali et al. estimates that $62 \%$ of the UAE's adults have a BMI $\geq 25 \mathrm{~kg} / \mathrm{m}^{2}$, based on data collected from five emirates in 2012, suggesting a slight drop in obesity and overweight rates [42]. In fact, the overall estimates of overweight and obesity reported by the study ( $42 \%$ and $20 \%$, respectively) [42] are clearly lower than those reported for earlier years [18]. However, it remains rather imprecise to deduce any trends in overweight or obesity rates by simply comparing the two studies due to their major differences with regard to design, sample recruitment, and specimen analysis. Nonetheless, the UAE still appears to be doing better than several of its neighbouring countries with regard to its overweight and obesity rates, such as Oman or Saudi Arabia (ASR of $64.7 \%$ and $63.6 \%$, respectively) $[48,49]$.

The World Health Organization's (WHO) more recent report on NCD prevalence demonstrates high rates of overweight and obesity in the UAE's adults, reporting similar estimates for the two sexes $(75.8 \%$ in female and $73.1 \%$ in male) [48]. Such national-level estimates in the UAE mirror the combined rates reported by one of our included studies for overweight and obesity in each sex among the Emirati adults residing in Abu Dhabi (overweight ASR: $34.4 \%$ in men and $33.6 \%$ in women, added to obesity ASR: $41.5 \%$ in men and $40.7 \%$ in women) [20]. This is possibly due to Abu Dhabi's (UAE capital) dense population and prominent role in the country's economic and political status, somewhat rendering it a miniature representative version of the entire country. The aforementioned study highlights that while the two sexes in the UAE have almost equal rates of overweight and obesity, their counterparts in the United States (US) have an almost 10\% difference in these rates ( $75 \%$ in men versus $66.5 \%$ in women) [50]. More extremely, Oman shows an almost 10-fold higher ASR for obesity in its women compared to men $(44.3 \%$ versus $4.7 \%$, respectively) [51]. This is likely because the rates of overweight and obesity across the two sexes remarkably differ by region, depending primarily on differences in social and cultural values, as highlighted in the 2011 global survey [52]. As for trending the rates of overweight and obesity in the UAE, only one study included in our review reports an observed uptrend of about $35 \%$ in the Emirati women residing in the Al Ain city of Abu Dhabi between 2000 and 2004, without reporting any trends for rates in men [34]. In fact, none of the included studies addresses the trends in overweight and obesity rates in men, another issue highlighted by the global survey as well [52].

As for the metabolic syndrome, only one included study addressed the national prevalence of metabolic syndrome among the UAE's adults between 1999 and 2000, reporting overall rates of $38 \%$ (as per NCEP definition) and $41 \%$ (IDF definition). The study also highlights remarkable differences in the sex-specific estimates of metabolic syndrome, reporting higher rates for women ( $47 \%$ and $46 \%$ as defined by the NCEP and IDF, respectively) compared to men (32\% and $33 \%$ as defined by the NCEP and IDF, respectively) [17]. In contrast, the overall prevalence of metabolic syndrome in Oman as defined by the IDF was only $19.8 \%$ in 2006 , almost half of that in the UAE [53]. Interestingly, however, the Omani study highlights even more pronounced differences in the sex-specific ASRs for metabolic syndrome (18.4\% in men versus $40 \%$ in women) [53] compared to those in the UAE. This clearly demonstrates much lower rates of metabolic syndrome in Omani males compared to their UAE counterparts (18.4\% versus 33\%), as opposed to the almost equal rates seen across Omani and UAE females (40\% and $46 \%$, respectively) [17]. In contrast, Saudi Arabia's overall ASR for metabolic syndrome was reported to be $39.3 \%$ between 1995 and 2000, as defined by NCEP [54], which is almost similar to the UAE's 1999-2000 estimate (41\%). Likewise, the sex-specific ASRs for metabolic syndrome reported for the two sexes in Saudi Arabia also mirror those of men and women in the UAE using the NCEP definition (37.2\% and $42 \%$ in Saudi Arabia's men and women, respectively, versus $32 \%$ and $47 \%$ in the UAE's men and women, respectively) [54].

Regarding dyslipidemia, there is an uptrend in the overall dyslipidemia rates in the UAE in recent years. A 2012 study included in our review addressed Emirati adults residing in Abu Dhabi and reported an ASR of $50.7 \%$ for 
dyslipidemia [20]. Another study conducted 3 years later reported a higher overall estimate of $68.5 \%$ for dyslipidemia prevalence among the adults in five of the UAE's emirates [42]. However, while the former study highlights remarkable differences in the sex-specific rates of dyslipidemia, reporting crude rates of $57.7 \%$ in men versus $33.9 \%$ in women [20], the more recent study does not break its overall estimate into sex-specific rates, making us unable to trend the sex-specific rates of dyslipidemia in recent years. In contrast, despite its higher rates of overweight and obesity, Saudi Arabia paradoxically has a lower overall rate of dyslipidemia (44\%) compared to the UAE (68.5\%), as reported by a national Saudi study published in the same year [55]. As for the sex-specific rates of the individual components of dyslipidemia in Saudi Arabia, the latter study reported similar rates of hypercholesterolemia and elevated low-density lipoprotein cholesterol (LDL-C) levels in both sexes (18.7\% and 30.7\% in men and $19.9 \%$ and $29.8 \%$ in women, respectively), with significantly higher rates of low HDL-C levels in men compared to women $(33.7 \%$ versus $17.7 \%$, respectively; $p$ value $=0.001)$ [55]. A national study conducted in Kuwait, another neighbouring country to the UAE, addressed the temporal trends in hypercholesterolemia rates defined according to the NCEP criteria from 1998 through 2009 [49]. The study clearly reported a progressive increase in the sexspecific rates of hypercholesterolemia, reaching $56 \%$ and $53.6 \%$ in men and women, respectively, in 2007 , followed by a significant drop thereafter to $33.7 \%$ and $30.6 \%$, respectively, in 2009 [49]. However, given that the prevalence rates of hypercholesterolemia, high LDL-C levels, or low HDL-C levels were collectively reported as "dyslipidemia" in the studies conducted in the UAE, we were unable to compare the specific rates of each of those lipid entities in the UAE to their corresponding estimates in nearby countries.

Only one included study addressed the prevalence of diabetes at the national level in the adult population of the UAE, reporting an overall ASR of $21 \%$ for diabetes and $7 \%$ for impaired fasting glucose between 1999 and 2000 [18]. These values are clearly higher than those reported in the US during the same time interval (1999-2000; 8.6\% and 6.2\%, respectively) [56], highlighting remarkably higher rates of diabetes in the UAE compared to the US ( $21 \%$ versus $8.6 \%$, respectively), despite similar rates of impaired fasting glucose (7\% versus $6.2 \%$, respectively). However, both studies report similar ASR of diabetes in men and women, although the overall rates reported in the UAE $(20.4 \%$ and $22.3 \%$, respectively) are higher than those seen in the US ( $9.3 \%$ and $8.1 \%$, respectively) [56]. In contrast, impaired fasting glucose rates are remarkably higher in women compared to men in the UAE ( $7.2 \%$ versus $4.5 \%$, respectively) [18], which is the complete opposite of what is seen in the US where the ASR for impaired fasting glucose in men is almost double that in women ( $8.3 \%$ versus $4.5 \%$, respectively) [56].

Furthermore, a more recent study included in our review reports ASR of $29.5 \%$ and $24.6 \%$ for prediabetes and diabetes, respectively. Taken together, these rates suggest that more than half of the Emirati adults of Abu Dhabi suffer from impaired glycemic control [20]. However, no differences were noted across the sex-specific rates reported by that study.
These findings should alarm public health policy-makers in the UAE and should highlight the need for prompt intervention to curb the high rates of impaired glycemic control in the UAE in order to prevent further progression into diabetes [57]. Additionally, it is important to draw attention to the 2011 IDF statistics which rank Kuwait first in the world with regard to its high national prevalence of type 2 diabetes (21.2\%), followed by Qatar (20.1\%), Saudi Arabia (20.0\%), and Bahrain (19.8\%), all of which happen to fall in the immediate vicinity of the UAE [58]. This clearly shows a regional pooling of diabetes along the western banks of the Arabian Gulf, possibly due to underlying common genetic and/or ethnic backgrounds of the citizens in those countries, added to the possible roles of their similar environmental, social, and dietary factors as well.

Hypertension also falls among the UAE's predominant cardiometabolic disorders, with all relevant studies conducted to date reporting emirate-specific estimates rather than national ones $[20,39,42]$. Thus, we refer to the WHO 2014 global status report on NCDs that found an overall ASR of $26.3 \%$ for elevated blood pressure in the UAE and almost similar rates across the two sexes $(27.5 \%$ in men and $23.3 \%$ in women) [48]. In contrast, Dubai Health Authority (DHA) and Dubai Statistics Center (DSC) collaboratively gathered data on the prevalence of various cardiovascular risk factors including hypertension between 2014 and 2016 and reported an overall estimate of $18.9 \%$ for hypertension prevalence among the Emirati citizens of Dubai [59]. The study also reports similar rates across the two sexes $(20 \%$ in men and $18 \%$ in women). Another emirate-specific study run by the Health Authority of Abu Dhabi (HAAD) 3 years earlier than the latter study reports an overall prevalence of $17 \%$ for hypertension among Abu Dhabi's Emirati citizens compared to double that prevalence (35\%) in its non-Emirati citizens [60]. However, contrary to the similar sex-specific rates of hypertension reported in the Dubai study [59], HAAD highlighted a significant difference in the rates of hypertension across the two sexes in Abu Dhabi, reporting a twofold higher rate in Emirati men (24\%) compared to women (12\%) [60]. Saudi Arabia shares similar estimates with its UAE neighbour, reporting an almost identical overall prevalence rate of hypertension among its adults (25.5\%) in 2011 [61]. In contrast, Oman, another country bordering the UAE, reports almost double that rate for adult hypertension, giving a strikingly high estimate of $41.5 \%$ in 2015 [62]. However, Yemen, a country bordering each of Saudi Arabia, Oman, and the UAE, reported a remarkably much lower overall ASR of hypertension than any of its neighbours in 2013 (7.7\%) [63]. Such findings pose a question on the factors that play a major role in the development of hypertension, given that its rates in geographically proximal, culturally similar, and ethnically related countries are remarkably different as shown previously. This also contradicts with the apparent pooling of diabetes in that same geographical area, as highlighted earlier.

Our review had some limitations worthy of being addressed. For instance, costs associated with cardiometabolic disorders in the UAE were not properly reported due to the lack of relevant studies. Moreover, the overall quality of some national studies limited our ability to 
provide conclusive evidence about the trend of cardiometabolic disorders.

\section{Conclusions}

However, our systematic review's major strength is its highly sensitive search strategy that possibly covered all relevant and intended literatures adequately. Moreover, our adherence to standardized and validated methods in conducting the review [10], our transparency in disseminating our search strategy (Appendix A in Supplementary Materials), and our use of standardized and previously tested data extraction and risk of bias assessment tools further support the review, increasing confidence in its reported findings. We are also the first team of researchers to conduct a systematic review of the epidemiology of obesity and cardiometabolic disorders in the UAE, setting grounds for subsequent researchers to build on. Thus, the ultimate aim of this review was to simply provide insights into the current prevalence rates and associated costs of cardiometabolic disorders in the UAE, making local decision-makers better informed and therefore capable of altering and tailoring future health policies accordingly.

$\begin{array}{ll}\text { Abbreviations } \\ \text { UAE: } & \text { United Arab Emirates } \\ \text { NCDs: } & \text { Noncommunicable diseases } \\ \text { PROSPERO: } & \text { International Prospective Register of } \\ & \text { Systematic Reviews } \\ \text { CINAHL: } & \text { Cumulative Index to Nursing and Allied } \\ & \text { Health Literature } \\ \text { IMEMR: } & \text { Index Medicus for the Eastern Mediterranean } \\ & \text { Region } \\ \text { PQDT: } & \text { ProQuest Dissertations \& Theses Database } \\ \text { OATD: } & \text { Open Access Theses and Dissertations } \\ \text { IDF: } & \text { International Diabetes Federation } \\ \text { COI: } & \text { Cost of illness } \\ \text { NCEP: } & \text { National Cholesterol Education Adult Panel } \\ & \text { III } \\ \text { HDL-C: } & \text { High-density lipoprotein cholesterol } \\ \text { IFG: } & \text { Impaired fasting glucose } \\ \text { ASR: } & \text { Age-standardized rate } \\ \text { PY: } & \text { Person-years } \\ \text { BMI: } & \text { Body mass index } \\ \text { WHO: } & \text { World Health Organization } \\ \text { US: } & \text { United States } \\ \text { LDL-C: } & \text { Low-density lipoprotein cholesterol } \\ \text { DHA: } & \text { Dubai Health Authority } \\ \text { DSC: } & \text { Dubai Statistics Center } \\ \text { HAAD: } & \text { Health Authority of Abu Dhabi. } \\ & \end{array}$

\section{Conflicts of Interest}

The authors declare that they have no conflicts of interest.

\section{Authors' Contributions}

$\mathrm{HR}$ and RR contributed to conception and design. HR, RB, $\mathrm{RR}$, and $\mathrm{HH}$ were involved in the title, abstract, and full-text screening. All authors were involved in analyzing and interpreting the data. $\mathrm{HR}, \mathrm{RB}$, and $\mathrm{RR}$ drafted the manuscript. $\mathrm{HH}, \mathrm{NL}$, and $\mathrm{MK}$ revised the manuscript and provided critical editing and comments. $H R, R B$, and $R R$ gave final approval of the version to be published. All authors reviewed and revised the draft manuscript and approved the submitted version.

\section{Acknowledgments}

We would like to extend our gratitude to Mrs. Nadia Masoud, Director of University of Sharjah Libraries, for her help in database search and retrieving full-text articles.

\section{Supplementary Materials}

Appendix A: search strategy followed and flow chart. Appendix B: quality assessment of the cost of illness for diabetes in the United Arab Emirates study. (Supplementary Materials)

\section{References}

[1] S. S. Lim, T. Vos, A. D. Flaxman et al., "A comparative risk assessment of burden of disease and injury attributable to 67 risk factors and risk factor clusters in 21 regions, 1990-2010: a systematic analysis for the Global Burden of Disease Study 2010," The Lancet, vol. 380, no. 9859, pp. 2224-2260, 2012.

[2] C. J. Henry, H. J. Lightowler, and H. M. Al-Hourani, "Physical activity and levels of inactivity in adolescent females ages 11-16 years in the United Arab Emirates," American Journal of Human Biology, vol. 16, no. 3, pp. 346-353, 2004.

[3] S. W. Ng, S. Zaghloul, H. Ali et al., "Nutrition transition in the United Arab Emirates," European Journal of Clinical Nutrition, vol. 65, no. 12, pp. 1328-1337, 2011.

[4] Y. Goryakin and M. Suhrcke, "Economic development, urbanization, technological change and overweight: what do we learn from 244 Demographic and Health Surveys?," Economics \& Human Biology, vol. 14, pp. 109-127, 2014.

[5] E. G. Wilmot, C. L. Edwardson, F. A. Achana et al., "Sedentary time in adults and the association with diabetes, cardiovascular disease and death: systematic review and meta-analysis," Diabetologia, vol. 55, no. 11, pp. 2895-2905, 2012.

[6] T. Loney, T. C. Aw, D. G. Handysides et al., "An analysis of the health status of the United Arab Emirates: the 'Big 4' public health issues," Global Health Action, vol. 6, no. 1, p. 20100, 2013.

[7] SMPP and N. Bo, Global Atlas on Cardiovascular Disease Prevention and Control, World Health Organization in Collaboration with the World Heart Federation and the World Stroke Organization, Geneva, Switzerland, 2011.

[8] UAE 2021 Vision, https://www.vision2021.ae/en/our-vision.

[9] B. Devleesschauwer, C. Maertens de Noordhout, G. S. Smit et al., "Quantifying burden of disease to support public health policy in Belgium: opportunities and constraints," $B M C$ Public Health, vol. 14, no. 1, p. 1196, 2014.

[10] D. Moher, A. Liberati, J. Tetzlaff, D. G. Altman, and P. Group, "Preferred reporting items for systematic reviews and metaanalyses: the PRISMA statement," International Journal of Surgery, vol. 8, no. 5, pp. 336-341, 2010.

[11] D. Hoy, P. Brooks, A. Woolf et al., "Assessing risk of bias in prevalence studies: modification of an existing tool and 
evidence of interrater agreement," Journal of Clinical Epidemiology, vol. 65, no. 9, pp. 934-939, 2012.

[12] F. Al-Maskari, M. El-Sadig, and N. Nagelkerke, "Assessment of the direct medical costs of diabetes mellitus and its complications in the United Arab Emirates," BMC Public Health, vol. 10, no. 1, p. 679, 2010.

[13] J. Sreedharan, J. Muttappallymyalil, S. Al Sharbatti et al., "Incidence of type 2 diabetes mellitus among Emirati residents in Ajman, United Arab Emirates," Korean Journal of Family Medicine, vol. 36, no. 5, pp. 253-257, 2015.

[14] F. Al-Haddad, Y. Al-Nuaimi, B. B. Little, and M. Thabit, "Prevalence of obesity among school children in the United Arab Emirates," American Journal of Human Biology, vol. 12, no. 4, pp. 498-502, 2000.

[15] M. Malik and A. Bakir, "Prevalence of overweight and obesity among children in the United Arab Emirates," Obesity Reviews, vol. 8, no. 1, pp. 15-20, 2007.

[16] Y. M. Abdulrazzaq, N. Nagelkerke, and M. A. Moussa, "UAE population reference standard charts for body mass index and skinfold thickness, at ages 0-18 years," International Journal of Food Sciences and Nutrition, vol. 62, no. 7, pp. 692-702, 2011.

[17] M. Malik and S. A. Razig, "The prevalence of the metabolic syndrome among the multiethnic population of the United Arab Emirates: a report of a national survey," Metabolic Syndrome and Related Disorders, vol. 6, no. 3, pp. 177-186, 2008.

[18] M. Malik, A. Bakir, B. A. Saab, and H. King, "Glucose intolerance and associated factors in the multi-ethnic population of the United Arab Emirates: results of a national survey," Diabetes Research and Clinical Practice, vol. 69, no. 2, pp. 188-195, 2005.

[19] R. M. Al-Mukhtar, "obesity in female students in the United Arab Emirates university," Bahrain Medical Bulletin, vol. 22, pp. 136-137, 2000.

[20] C. Hajat, O. Harrison, and Z. Al Siksek, "Weqaya: a population-wide cardiovascular screening program in Abu Dhabi, United Arab Emirates," American Journal of Public Health, vol. 102, no. 5, pp. 909-914, 2012.

[21] H. M. Al-Hourani, C. J. Henry, and H. J. Lightowler, "Prevalence of overweight among adolescent females in the United Arab Emirates," American Journal of Human Biology, vol. 15, no. 6, pp. 758-764, 2003.

[22] F. H. Al-Haddad, B. B. Little, and A. G. Abdul Ghafoor, "Childhood obesity in United Arab Emirates schoolchildren: a national study," Annals of Human Biology, vol. 32, no. 1, pp. 72-79, 2005.

[23] A. Al Blooshi, S. Shaban, M. AlTunaiji et al., "Increasing obesity rates in school children in United Arab Emirates," Obesity Science \& Practice, vol. 2, no. 2, pp. 196-202, 2016.

[24] A. A. Bin Zaal, A. O. Musaiger, and R. D’Souza, "Dietary habits associated with obesity among adolescents in Dubai, United Arab Emirates," Nutrición Hospitalaria, vol. 24, no. 4, pp. 437-444, 2009.

[25] A. Al Junaibi, A. Abdulle, S. Sabri, M. Hag-Ali, and N. Nagelkerke, "The prevalence and potential determinants of obesity among school children and adolescents in Abu Dhabi, United Arab Emirates," International Journal of Obesity, vol. 37, no. 1, pp. 68-74, 2013.

[26] A. O. Musaiger, M. Al-Mannai, R. Tayyem et al., "Prevalence of overweight and obesity among adolescents in seven Arab countries: a cross-cultural study," Journal of Obesity, vol. 2012, Article ID 981390, 5 pages, 2012.
[27] E. K. Amine and M. Samy, "Obesity among female university students in the United Arab Emirates," Journal of the Royal Society of Health, vol. 116, no. 2, pp. 91-96, 1996.

[28] H. E. Badr and F. El-Sabban, "Body mass index and type 1 diabetes mellitus in United Arab Emirates University students: a pilot study," Emirates Medical Journal, vol. 26, no. 1, pp. 31-33, 2008.

[29] A. O. Musaiger, O. L. Lloyd, S. M. Al-Neyadi, and A. B. Bener, "Lifestyle factors associated with obesity among male university students in the United Arab Emirates," Nutrition \& Food Science, vol. 33, no. 4, pp. 145-147, 2003.

[30] L. I. Sheikh-Ismail, C. J. Henry, H. J. Lightowler, A. S. Aldhaheri, E. Masuadi, and H. M. Al Hourani, "Prevalence of overweight and obesity among adult females in the United Arab Emirates," International Journal of Food Sciences and Nutrition, vol. 60, no. 3, pp. 26-33, 2009.

[31] A. Kerkadi, "Evaluation of nutritional status of United Arab Emirates University female students," Emirates Journal of Food and Agriculture, vol. 15, no. 2, pp. 42-50, 2003.

[32] A. O. Musaiger and H. M. Radwan, "Social and dietary factors associated with obesity in university female students in United Arab Emirates," Journal of the Royal Society of Health, vol. 115, no. 2, pp. 96-99, 1995.

[33] D. Papandreou, Z. T. Noor, M. Rashed, and H. A. Jaberi, "Association of neck circumference with obesity in female college students," Open Access Macedonian Journal of Medical Sciences, vol. 3, no. 4, pp. 578-581, 2015.

[34] A. O. Carter, H. F. Saadi, R. L. Reed, and E. V. Dunn, "Assessment of obesity, lifestyle, and reproductive health needs of female citizens of Al Ain, United Arab Emirates," Journal of Health, Population and Nutrition, vol. 22, no. 1, pp. 75-83, 2004.

[35] A. E. Mehairi, A. A. Khouri, M. M. Naqbi et al., "Metabolic syndrome among Emirati adolescents: a school-based study," PLoS One, vol. 8, no. 2, Article ID e56159, 2013.

[36] A. S. Al Dhaheri, M. N. Mohamad, A. H. Jarrar et al., "A crosssectional study of the prevalence of metabolic syndrome among young female Emirati adults," PLoS One, vol. 11, no. 7, Article ID e0159378, 2016.

[37] M. M. Agarwal, P. F. Hughes, A. A. Haliga, P. Newman, M. M. Sheekh-Hussen, and A. G. Shalabi, "Relevance of cholesterol screening in the United Arab Emirates: a preliminary study," European Journal of Epidemiology, vol. 11, no. 5, pp. 581-585, 1995.

[38] A. Abdulle, A. Al-Junaibi, and N. Nagelkerke, "High blood pressure and its association with body weight among children and adolescents in the United Arab Emirates," PLoS One, vol. 9, no. 1, Article ID e85129, 2014.

[39] Y. I. El-Shahat, S. Z. Bakir, N. Farjou et al., "Hypertension in UAE citizens-preliminary results of a prospective study," Saudi Journal of Kidney Diseases and Transplantation, vol. 10, no. 3, pp. 376-381, 1999.

[40] I. T. El Mugamer, A. S. Ali Zayat, M. M. Hossain, and R. N. Pugh, "Diabetes, obesity and hypertension in urban and rural people of bedouin origin in the United Arab Emirates," Journal of Tropical Medicine and Hygiene, vol. 98, no. 6, pp. 407-415, 1995.

[41] H. Saadi, S. G. Carruthers, N. Nagelkerke et al., "Prevalence of diabetes mellitus and its complications in a population-based sample in Al Ain, United Arab Emirates," Diabetes Research and Clinical Practice, vol. 78, no. 3, pp. 369-377, 2007.

[42] A. Yusufali, N. Bazargani, K. Muhammed et al., "Opportunistic screening for CVD risk factors: the Dubai shopping for 
cardiovascular risk study (DISCOVERY)," Global Heart, vol. 10, no. 4, pp. 265-272, 2015.

[43] L. M. Baynouna, A. D. Revel, N. J. Nagelkerke et al., "High prevalence of the cardiovascular risk factors in Al-Ain, United Arab Emirates: an emerging health care priority," Saudi Medical Journal, vol. 29, no. 8, pp. 1173-1178, 2008.

[44] C. Hajat and O. Harrison, "The Abu Dhabi cardiovascular program: the continuation of Framingham," Progress in Cardiovascular Diseases, vol. 53, no. 1, pp. 28-38, 2010.

[45] M. M. Hossain and M. Malik, "Prevalences and correlates of diabetes, obesity, and hyperlipidemia in the United Arab Emirates (UAE)," Bahrain Medical Bulletin, vol. 20, no. 3, pp. 119-122, 1998.

[46] M. S. Newson-Smith, "Importing health conditions of expatriate workers into the United Arab Emirates," Asia Pacific Journal of Public Health, vol. 22, no. 3, pp. 25S-30S, 2010.

[47] K. M. Flegal, M. D. Carroll, C. L. Ogden, and C. L. Johnson, "Prevalence and trends in obesity among US adults, 19992000,” JAMA, vol. 288, no. 14, pp. 1723-1727, 2002.

[48] World Health Organization, Global Status Report on Noncommunicable Diseases 2014, World Health Organization, Geneva, Switzerland, 2014.

[49] H. G. Ahmed, I. A. Ginawi, A. M. Elasbali, I. M. Ashankyty, and A. M. Al-Hazimi, "Prevalence of obesity in Hail region, KSA: in a comprehensive survey," Journal of Obesity, vol. 2014, Article ID 961861, 5 pages, 2014.

[50] L. Yang and G. A. Colditz, "Prevalence of overweight and obesity in the United States, 2007-2012," JAMA Internal Medicine, vol. 175, no. 8, pp. 1412-1413, 2015.

[51] J. A. Al-Lawati, A. J. Mohammed, H. Q. Al-Hinai, and P. Jousilahti, "Prevalence of the metabolic syndrome among Omani adults," Diabetes Care, vol. 26, no. 6, pp. 1781-1785, 2003.

[52] R. Kanter and B. Caballero, "Global gender disparities in obesity: a review," Advances in Nutrition, vol. 3, no. 4, pp. 491-498, 2012.

[53] J. A. Al-Lawati and P. Jousilahti, "Prevalence of metabolic syndrome in Oman using the international diabetes federation's criteria," Saudi Medical Journal, vol. 27, no. 12, pp. 1925-1926, 2006.

[54] M. Al-Nozha, A. Al-Khadra, M. R. Arafah et al., "Metabolic syndrome in Saudi Arabia," Saudi Medical Journal, vol. 26, no. 12, pp. 1918-1925, 2005.

[55] A. A.-H. , N. Alkaabba, A. Tahir, A. Abdalla, G. Hussein, A. Saeed, and M. A. Hamza, "Prevalence and correlates of dyslipidemia among adults in Saudi Arabia: results from a national survey," Open Journal of Endocrine and Metabolic Diseases, vol. 2, no. 89, 2012.

[56] CDC Diabetes Program, National diabetes fact sheet: national estimates on diabetes, http://www.cdc.gov/diabetes/pubs/ factsheet.htm

[57] A. G. Tabak, C. Herder, W. Rathmann, E. J. Brunner, and M. Kivimaki, "Prediabetes: a high-risk state for diabetes development," The Lancet, vol. 379, no. 9833, pp. 2279-2290, 2012.

[58] M. Badran and I. Laher, "Type II diabetes mellitus in Arabicspeaking countries," International Journal of Endocrinology, vol. 2012, Article ID 902873, 11 pages, 2012.

[59] Announcing Dubai Health Survey Results 2016, https:// www.dsc.gov.ae/en-us/DSC-News/Pages/Dubai-HealthSurvey-Results2016.aspx.

[60] HAAD releases 2012 Health Statistics and Capacity Master Plan, https://haad.ae/HAAD/tabid/58/ctl/Details/Mid/417/ ItemID/379/Default.aspx.
[61] A. A. Saeed, N. A. Al-Hamdan, A. A. Bahnassy, A. M. Abdalla, M. A. Abbas, and L. Z. Abuzaid, "Prevalence, awareness, treatment, and control of hypertension among Saudi adult population: a national survey," International Journal of Hypertension, vol. 2011, Article ID 174135, 8 pages, 2011.

[62] M. A. Abd El-Aty, F. A. Meky, M. M. Morsi, J. A. Al-Lawati, and M. K. El Sayed, "Hypertension in the adult Omani population: predictors for unawareness and uncontrolled hypertension," Journal of the Egyptian Public Health Association, vol. 90, no. 3, pp. 125-132, 2015.

[63] P. A. Modesti, M. Bamoshmoosh, S. Rapi, L. Massetti, D. AlHidabi, and H. Al Goshae, "Epidemiology of hypertension in Yemen: effects of urbanization and geographical area," $\mathrm{Hy}$ pertension Research, vol. 36, no. 8, pp. 711-717, 2013. 


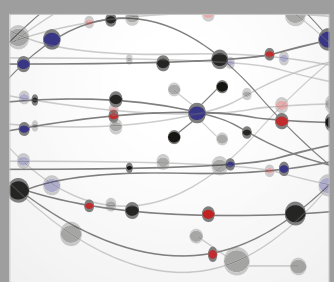

The Scientific World Journal
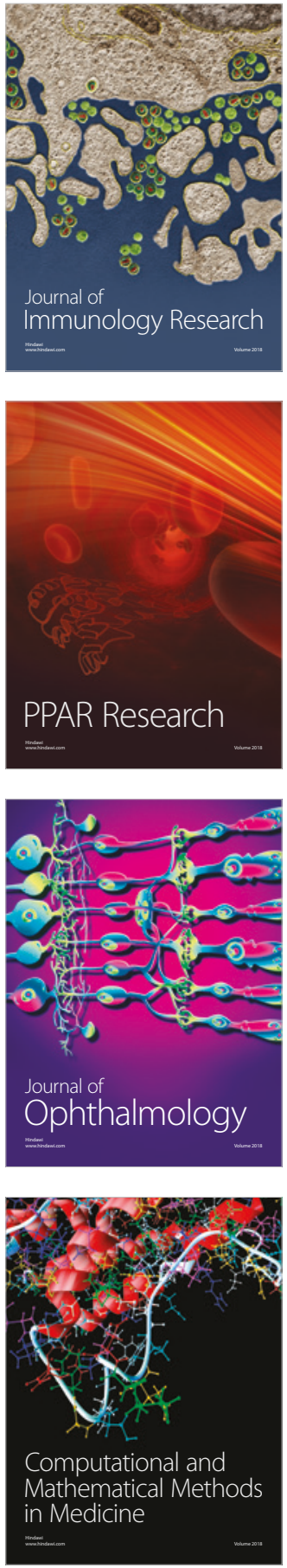

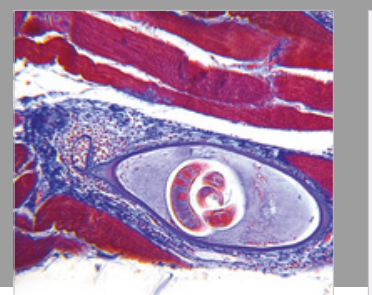

Gastroenterology Research and Practice

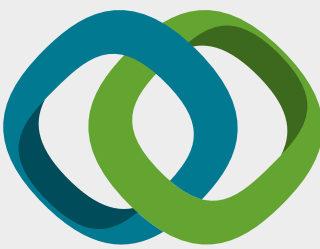

\section{Hindawi}

Submit your manuscripts at

www.hindawi.com
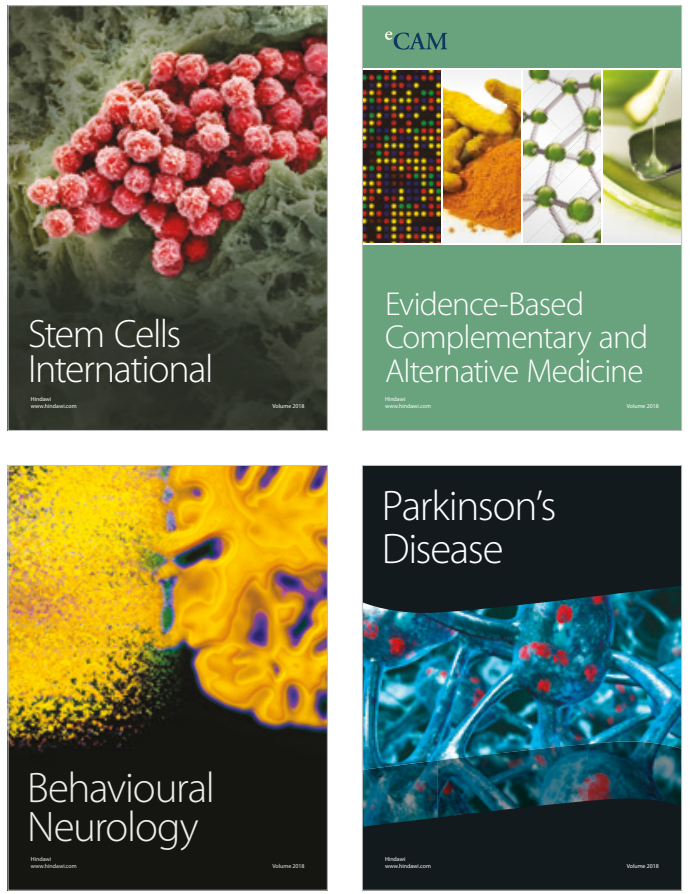

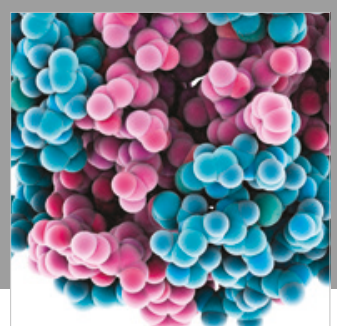

ournal of

Diabetes Research

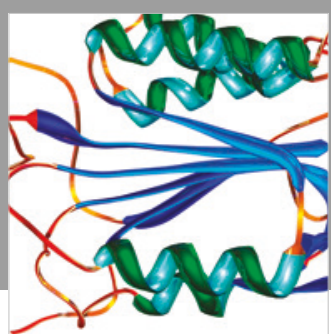

Disease Markers
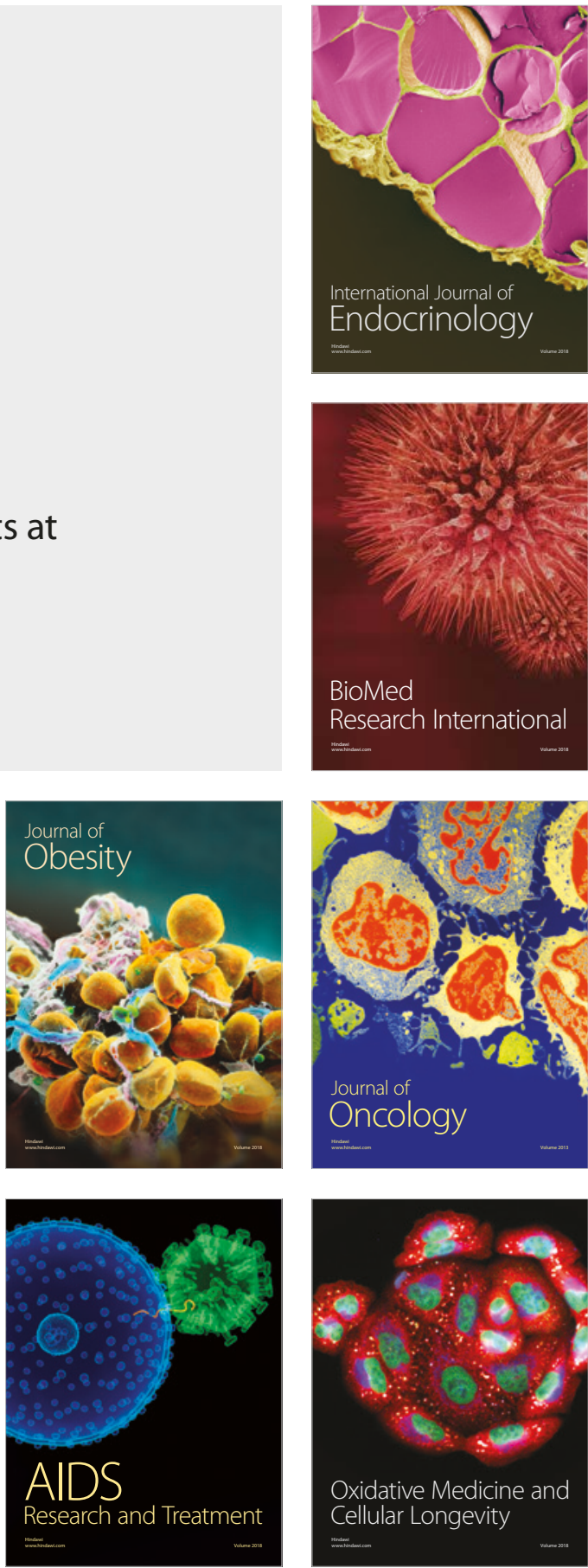